\title{
Design, analysis, and control of a cable-driven parallel platform with a pneumatic muscle active support \\ Xingwei Zhao $\dagger,{ }^{*}$ Bin $\mathrm{Zi} \ddagger$ and Lu Qian $\S$
}

\author{
$\dagger$ Chair of Mechatronics and Machine Dynamics, Technical University of Berlin, 10587, Berlin, \\ Germany \\ $\ddagger$ School of Mechanical and Automotive Engineering, Hefei University of Technology, 230009, Hefei, \\ P. R. China. \\ §Institute of Automatic Control and Complex Systems, University of Duisburg-Essen, 47057, \\ Duisburg, Germany.E-mails: binzi.cumt@163.com,qianluzxw@gmail.com
}

(Accepted September 17, 2015. First published online: October 19, 2015)

\begin{abstract}
SUMMARY
The neck is an important part of the body that connects the head to the torso, supporting the weight and generating the movement of the head. In this paper, a cable-driven parallel platform with a pneumatic muscle active support (CPPPMS) is presented for imitating human necks, where cable actuators imitate neck muscles and a pneumatic muscle actuator imitates spinal muscles, respectively. Analyzing the stiffness of the mechanism is carried out based on screw theory, and this mechanism is optimized according to the stiffness characteristics. While taking the dynamics of the pneumatic muscle active support into consideration as well as the cable dynamics and the dynamics of the Up-platform, a dynamic modeling approach to the CPPPMS is established. In order to overcome the flexibility and uncertainties amid the dynamic model, a sliding mode controller is investigated for trajectory tracking, and the stability of the control system is verified by a Lyapunov function. Moreover, a PD controller is proposed for a comparative study. The results of the simulation indicate that the sliding mode controller is more effective than the PD controller for the CPPPMS, and the CPPPMS provides feasible performances for operations under the sliding mode control.
\end{abstract}

KEYWORDS: Neck; Cable-driven parallel platform; Pneumatic muscle; Stiffness; Dynamics; Sliding mode control.

\section{Introduction}

Currently, there is a great deal of interest in investigations of the structure of the human body. ${ }^{1,2}$ The neck is an important part of the human body that connects the head to the torso, supporting the weight and generating movements of the head. Clinical studies show that the head motion relies on the cervical portion of the human spine which consists of seven cervical vertebraes. ${ }^{3}$ Although each vertebra has six-degrees-of-freedom (DOF), the neck is usually treated as a 3-DOF mechanism (i.e. pitch, roll, and yaw). Over the past decade, many mechanisms for simulating the neck's motion have been designed, which can be classified into two categories: the serial structure and the parallel structure.

The mechanism belonging to the serial structure is easier to control, since each DOF of the neck is actuated independently. The robots such as Honda ASIMO ${ }^{4} \mathrm{HRP}-3,{ }^{5}$ and $\mathrm{BHR}^{6}$ have serial necks with 2-DOF in terms of pitch and roll. LOLA, ${ }^{7}$ Dav $^{8}$ and Robota ${ }^{9}$ have serial necks with 3 -DOF in terms of pitch, roll, and yaw. ARMAR-III ${ }^{10}$ and WABIANRIV ${ }^{11}$ have serial necks with 4-DOF, where an additional DOF for nodding is considered.

\footnotetext{
* Corresponding author. E-mail: zhaoxingwei001@gmail.com
} 
However, it is necessary to note that the mechanisms that use the serial structure have different biological structures compared to that of the human neck. In the light of the biological structure of the human body, more and more humanoid necks have been designed with the parallel structure. An anthropomimetic robot was proposed by Holland. ${ }^{12}$ The neck of the robot is made up of four vertebraes driven by shock cords. However, the vertebraes in the robot are much longer than the vertebrases in humans so that the robot vertebraes can accommodate all of the motors and the tendons. A human-like tendon-driven neck was proposed by Nori et al. ${ }^{13,14}$ The neck bone of the mechanism is composed of a steel spring surrounded by steel tendons, but this structure has only 2-DOF in terms of pitch and roll. A cable-driven flexible parallel robot with low motion noise to mimic a human neck was presented by Gao et al. ${ }^{15}$ which is similar to the human-like tendon-driven neck in ref. [13]. Lee ${ }^{16}$ introduced a biomechanical model of the human head-neck system, which consists of 7 cervical vertebraes coupled with 3-DOF joints and 72 neck muscles arranged in 3 muscle layers. However, this biomechanical model is difficult to construct in reality due to the complexity of the structure. Furthermore, Liem et al. ${ }^{17}$ presented a Hexaspine by using the parallel structure to imitate the human spine, in which only one vertebra was concerned. From the literature mentioned above, great efforts have been made with regards to designing the humanoid neck. However, there is still a demand for a novel structure to imitate the human neck, which will facilitate the in-depth understanding of the motion of the neck.

In this paper, a CPPPMS is proposed as an optimal mechanism for imitating the human neck. The CPPPMS is a parallel mechanism with 4-DOF, in terms of pitch, roll, yaw, and has one additional translation DOF that describes the length of the pneumatic muscle active support. The pitch, roll, and yaw motion represent the head nod, head twisting, and head shaking, respectively. The additional translation DOF is used for absorbing the vertical vibration of the head. The major innovation within this mechanism is that the cable actuators and the pneumatic muscle actuator are utilized simultaneously. The cables driven system has a simple and lightweight structure, a large reachable workspace, and a high acceleration capability $;{ }^{18-21}$ while the pneumatic muscle has a high mass/weight ratio, a high flexibility, and has similarities to biological muscles. ${ }^{22-27}$ Therefore, the cable actuators imitate neck muscles due to the demand of the large length change, and the pneumatic muscle actuator imitates spinal muscles because of the large force requirement. In the author's previous work, ${ }^{22}$ a pneumatic muscle driven parallel mechanism for imitating human pelvis was investigated, where four pneumatic muscles are used to drive 4-DOF. However, this mechanism can only be fully controlled with a help of gravity. Its workspace, stiffness, and maximum acceleration are limited in small region. This mechanism can be utilized for rehabilitation of human pelvis but is not suitable for imitating the neck motion. Therefore, in order to make sure that the CPPPMS is controllable during required postures as well as with certain stiffness, five cables are used to control three rotary DOF and a pneumatic muscle controls one translation DOF, and the CPPPMS is thus a redundant drive system. ${ }^{21}$

As the CPPPMS is driven by flexible actuators, some uncertainties can be brought into the dynamics. Therefore, a sliding mode controller, which has advantages in attacking the uncertainties of a system, ${ }^{28}$ is used to control the CPPPMS. In this paper, a sliding mode controller is redesigned to adapt to the CPPPMS, which has a redundant drive system and has flexible actuators. Yong et al. ${ }^{29}$ proposed a non-singular terminal sliding mode control for rigid manipulators. This control is suitable for a second-order nonlinear dynamic system with parameters uncertainties and external disturbances, but the author did not mention the way to deal with flexible manipulators. A sliding mode control of a pneumatic muscle actuated servo system was proposed by Shen..$^{30}$ A hybrid control combining both a sliding mode control and an adaptive fuzzy CMAC uncertainty compensator was presented by Shi and Shen, ${ }^{31}$ where a parallel platform based on pneumatic muscles is controlled. However, the systems shown in refs. [30, 31] are non-redundant systems, how to use a sliding mode control for a redundant system needs to be further studied and accounted for.

The rest of the paper is organized as follows: Section 2 describes the design model of the mechanism. In Section 3, the stiffness characteristics of the mechanism are analyzed based on screw theory. By using the stiffness characteristics as index, the dimensions of the mechanism are optimized and the boundary of the real workspace is constructed. Subsequently, by taking the dynamics of the Up-platform, the cable dynamics, and the dynamics of the pneumatic muscle active support into consideration, a dynamic modeling approach to the mechanism is established in Section 4. A sliding mode controller is presented and its stability is verified by a Lyapunov function in Section 5. 

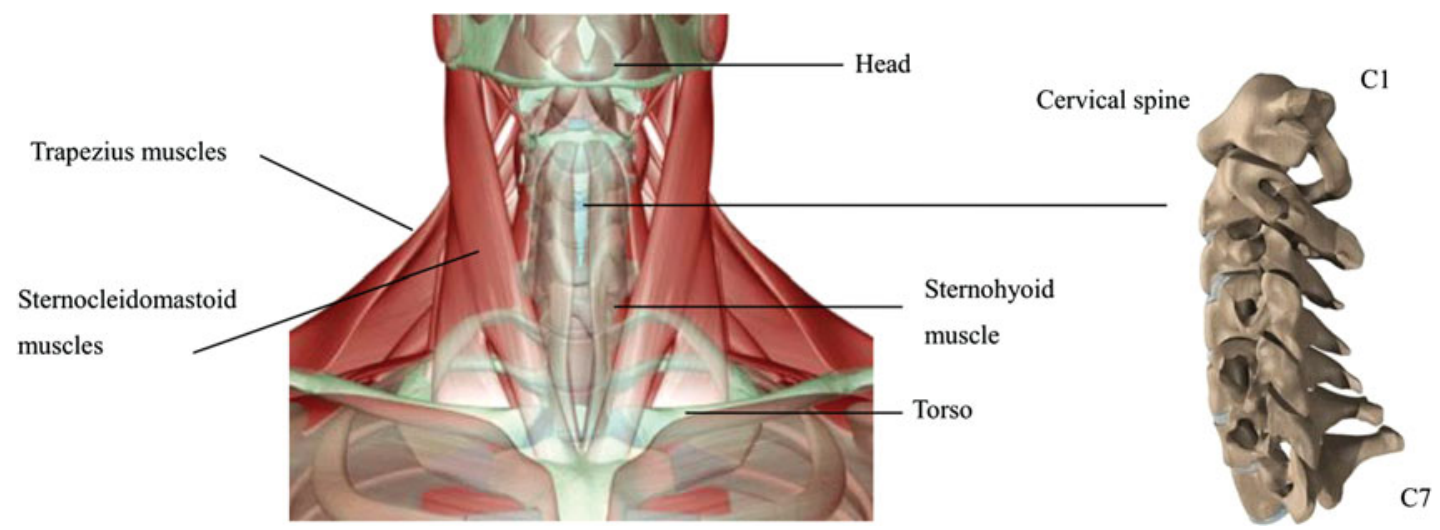

Fig. 1. Structure of the human neck.

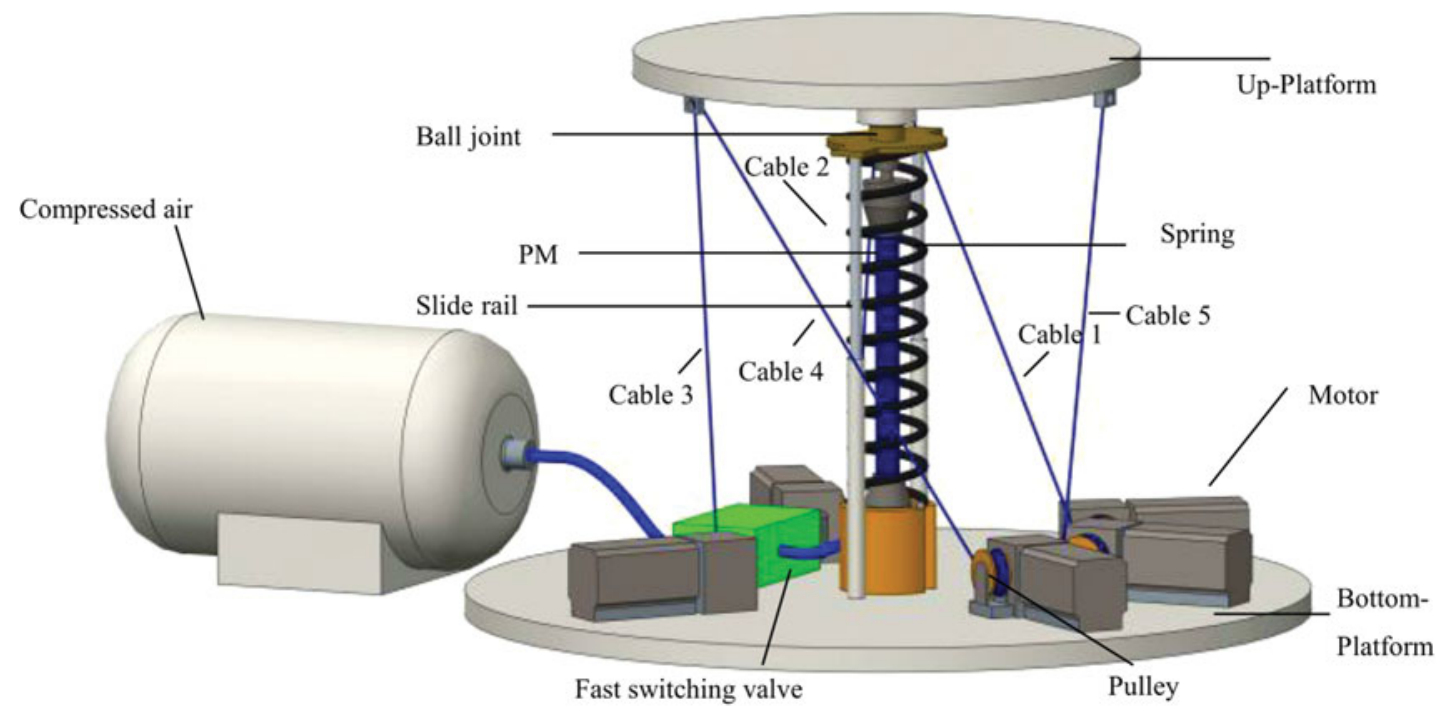

Fig. 2. Structure of the CPPPMS.

Meanwhile, a PD controller is proposed for a comparison to the study. In Section 6, the effectiveness of the sliding mode controller is compared with the PD controller by numerical simulations before the conclusions are drawn in Section 7.

\section{System Description}

The human neck is flexible and allows the head to turn and to flex in all directions. It consists of the cervical spine and muscles. The cervical spine comprises seven bony segments, typically referred to $\mathrm{C} 1$ to $\mathrm{C} 7$. When viewed from the side, an adult spine has a natural S-shaped curve. The curve works like a coiled spring to absorb shock, maintain balance, and allow range of motion throughout the spinal column. There are more than twenty types of muscles in a neck, and individual muscles often have multiple origins and insertions. Since it would be difficult to model all the muscles accurately, we are motivated to reduce the number of modeled muscles and consider only major muscles, which are the sternohyoid muscles, the sternocleidomastoid muscles, and the trapezius muscles, as shown in Fig. 1.

The structure of the CPPPMS is presented in Fig. 2. The Bottom-platform imitates the torso; whereas the Up-platform represented the head. The pneumatic muscle, spring, and slide rail form a pneumatic muscle active support, which imitates a neck spine with the S-shaped curve and the muscles. The five cables serve as neck muscles, where the cables Nr. 1 and Nr. 2 represent the right 


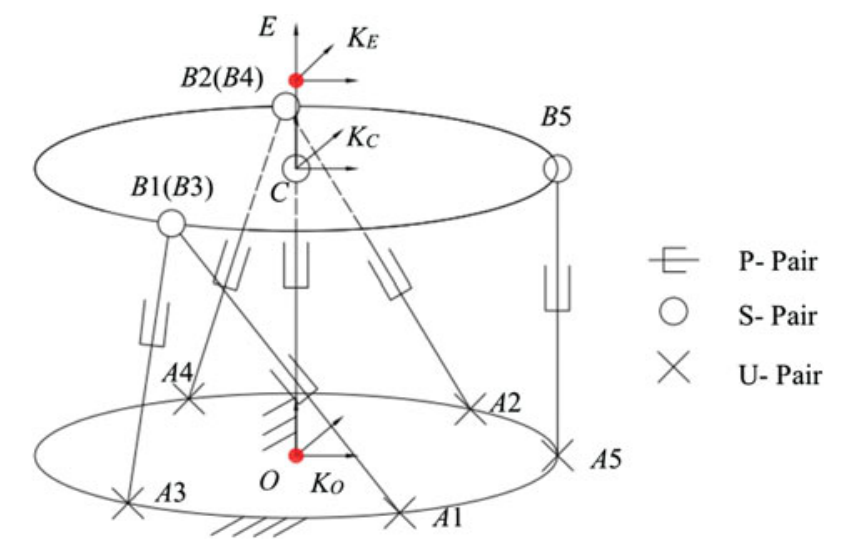

Fig. 3. Mechanism scheme of the CPPPMS (P represents a prismatic joint, S represents a spherical joint, U represents a universal joint).

and left sternocleidomastoid muscles, the cables Nr. 3 and Nr. 4 represent the right and left trapezius muscles, and the cable Nr. 5 represents the sternohyoid muscles, respectively.

\section{Stiffness Analysis and Dimensions Optimization}

In this section, the stiffness characteristics of the CPPPMS are analyzed. As a redundant driven mechanism, the index based on algebraic characteristics of Jacobian matrix is no more suitable for dimension optimization, because Jacobian matrix is not a square matrix. Therefore, the index based on stiffness characteristics is utilized for the dimensions optimization of the CPPPMS, which can identify singular positions as well as estimate the stiffness of the redundant driven mechanism under each configuration.

\subsection{Stiffness analysis}

The mechanism scheme of the CPPPMS is shown in Fig. 3. The pneumatic muscle active support can be treated as a PS-chain (P represents a prismatic joint, $\mathrm{S}$ represents a spherical joint), where two translation DOF are restrained. Each cable can be treated as a UPS-chain (U represents a universal joint) and no DOF is restricted. Therefore, the Up-platform has 4-DOF in terms of one translation and three rotations. There are five cables driven by motors and one pneumatic muscle driven by compressed air. In conclusion, the CPPPMS is a redundant driven mechanism with 4-DOF.

In order to describe the mechanism conveniently, some labels for each joint and the center of mass are given as follows. One side of the $\operatorname{Nr}$. $i$ th $(i=1,2,3,4,5)$ cable is connected to the $i$ th roller and passes through the Bottom-platform at the point $A_{i}$. The other side of the $i$ th cable is connected to the Up-platform at the point $B_{i}$. The pneumatic muscle active support is located between the center of the Bottom-platform $O$ and the center of the Up-platform $C$. A global coordinate $K_{O}$ is attached to the Bottom-platform, with its origin at the point $O$. Its $x$-axis is along the vector $O A_{5}$, and its $z$-axis is along the vector $O C$. In this paper, all coordinates are right hand coordinates and the $y$-axis is unique determined according to the right hand law. A coordinate $K_{C}$ is attached to the Up-platform, with its origin at the point $C$. Its $x$-axis is along the vector $C B_{5}$, and its $z$-axis is along the vector $C E$. The end-effector of the Up-platform is labeled as $E$, where a coordinate $K_{E}$ is set. $K_{E}$ is parallel with $K_{C}$.

In order to ensure enough space for assembling electric motors, five motors are equally spaced at 72 degree on the Bottom-platform. Therefore, the points of the intersection between the cables and the Bottom-platform $A_{i}(i=1,2,3,4,5)$ in coordinate $K o$ are described by vector $\boldsymbol{r}_{O A i}$,

$$
\boldsymbol{r}_{O A i}=\left[R_{a} \cos \phi_{i}, R_{a} \sin \phi_{i}, 0\right]^{T}
$$

where $R_{a}$ is the radius of the Bottom-platform, and $\phi_{i}$ are given as

$$
\phi_{1}=72^{\circ}, \quad \phi_{2}=-72^{\circ}, \quad \phi_{3}=144^{\circ}, \quad \phi_{4}=-144^{\circ}, \quad \phi_{5}=0^{\circ} .
$$


From Fig. 1 one can see that the connected points between the muscles and the head are not equally but symmetrically spaced. One of the reasons is that the neck needs to avoid singular positions in its workspace. The locations of the joints between the cables and the Up-platform $B_{i}(i=1,2,3,4,5)$ in coordinate $K c$ are described by the vector $\boldsymbol{r}_{C B i}$,

$$
\boldsymbol{r}_{C B i}=\left[R_{b} \cos \psi_{i}, R_{b} \sin \psi_{i}, 0\right]^{T},
$$

where $R_{b}$ is the radius of the Up-platform, and $\psi_{i}$ is given by

$$
\psi_{1}=-\psi_{2}=v_{1}, \quad \psi_{3}=-\psi_{4}=v_{2}, \quad \psi_{5}=0^{\circ},
$$

where $v_{1}$ and $v_{2}$ are two unknown variables which will be optimized later.

The vector $\boldsymbol{r}_{O C}$ from the point $O$ to $C$, which describes the vector of pneumatic muscle active support in the coordinate $K_{O}$, is given as

$$
\boldsymbol{r}_{O C}=\left[\begin{array}{lll}
0 & 0 & h_{1}
\end{array}\right]^{\mathrm{T}},
$$

where $h_{1}$ is the length of the pneumatic muscle active support. Unless otherwise specified, hereafter the subscripts of all the variables are described in the coordinate $K_{O}$.

The vector $\boldsymbol{r}_{A B i}$ which describes the $i$ th cables in coordinate $K o$ is given as

$$
\boldsymbol{r}_{A B i}=-\boldsymbol{r}_{O A i}+\boldsymbol{r}_{O C}+\boldsymbol{R} \cdot \boldsymbol{r}_{C B i}, \quad i=1,2,3,4,5,
$$

where $\boldsymbol{R}$ is the orientation matrix of the Up-platform by introducing yaw, pitch, and roll angles $(\alpha, \beta, \gamma)$,

$$
\boldsymbol{R}=\left[\begin{array}{ccc}
C \alpha C \beta & C \alpha S \beta S \gamma-S \alpha C \gamma & C \alpha S \beta C \gamma+S \alpha S \gamma \\
S \alpha C \beta & S \alpha S \beta S \gamma+C \alpha C \gamma & S \alpha S \beta C \gamma-C \alpha S \gamma \\
-S \beta & C \beta S \gamma & C \beta C \gamma
\end{array}\right],
$$

where $C$ denotes cosine and $S$ denotes sine.

The length of the $i$ th cable is

$$
S_{A B i}=\left|\boldsymbol{r}_{A B i}\right| .
$$

The unit vectors along the direction of $\boldsymbol{r}_{A B i}$ and $\boldsymbol{r}_{O C}$ are given as

$$
\boldsymbol{s}_{A B i}=\boldsymbol{r}_{A B i} /\left|\boldsymbol{r}_{A B i}\right|, \quad \boldsymbol{s}_{O C}=\boldsymbol{r}_{O C} /\left|\boldsymbol{r}_{O C}\right| .
$$

The stiffness characteristics of the CPPPMS are analyzed by screw theory. Compared with other methods, screw theory is simple and brief for stiffness modeling. ${ }^{32,33}$ The pneumatic muscle active support is a PS-chain, which provides two restrained wrenches $\hat{\boldsymbol{\phi}}_{w c 1}$ and $\hat{\boldsymbol{\$}}_{w c 2}$ representing the forces on the $x$ - and $y$ - direction and one driven wrench $\hat{\mathbf{S}}_{\text {wap }}$ representing the force on the $z$-direction. Each cable offers one driven wrench $\hat{\$}_{w a i}(i=1,2,3,4,5)$ representing the force along each axis of the cables. Ignoring gravity and friction, the wrench $\hat{\$}_{w}$ acting on the Up-platform is provided by each chain. Therefore, the base space of the wrench $\boldsymbol{W}$ based on screw theory is

$$
\boldsymbol{W}=\left[\begin{array}{llllllll}
\hat{\mathbf{\$}}_{w a 1} & \hat{\mathbf{\$}}_{w a 2} & \hat{\mathbf{\$}}_{w a 3} & \hat{\mathbf{\$}}_{w a 4} & \hat{\mathbf{\$}}_{w a 5} & \hat{\mathbf{\$}}_{w a p} & \hat{\mathbf{\$}}_{w c 1} & \hat{\mathbf{\$}}_{w c 2}
\end{array}\right],
$$

here

$$
\begin{gathered}
\hat{\mathbf{\$}}_{w a 1}=\left[\begin{array}{c}
\boldsymbol{s}_{A B i} \\
\boldsymbol{r}_{C B i} \times \boldsymbol{s}_{A B i}
\end{array}\right], \quad i=1,2,3,4,5 \\
\hat{\mathbf{\$}}_{\text {wap }}=[0,0,1,0,0,0]^{T}, \quad \hat{\boldsymbol{\$}}_{w c 1}=[1,0,0,0,0,0]^{T}, \quad \hat{\mathbf{\$}}_{w c 2}=[0,1,0,0,0,0]^{T} .
\end{gathered}
$$


Table I. Algorithm to calculate the stiffness characteristics.

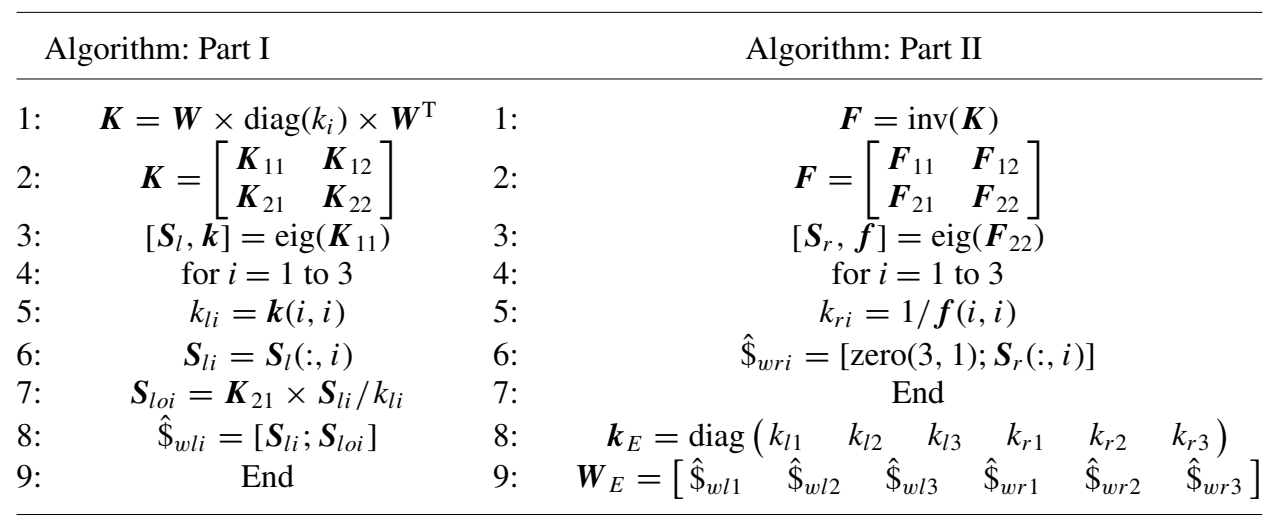

The stiffness of the cables is assumed as $k_{a}$, whose calculation approach is proposed in Section 4.2. The stiffness of the pneumatic muscle active support is approximately equivalent to the stiffness of the spring $k_{s}$. Compared with the cables and the pneumatic muscle, the stiffness of constraint joint is approaching to infinite, which is assumed as $k_{c}$. The stiffness of each chain is written in the form of diagonal matrix,

$$
\boldsymbol{k}=\operatorname{diag}\left(k_{a 1} \quad k_{a 2} \quad k_{a 3} \quad k_{a 4} \quad k_{a 5} \quad k_{s} \quad k_{c 1} \quad k_{c 2}\right)
$$

Once the wrench and stiffness of each chain are known, the stiffness characteristics of the CPPPMS can be analyzed by the decomposition of eigen-twists and eigen-wrenches, ${ }^{34,35}$

$$
\boldsymbol{W}_{E} \boldsymbol{k}_{E} \boldsymbol{W}_{E}^{\mathrm{T}}=\boldsymbol{W} k W^{\mathrm{T}}
$$

here

$$
\begin{aligned}
& \boldsymbol{W}_{E}=\left[\begin{array}{llllll}
\hat{\boldsymbol{\$}}_{w l 1} & \hat{\boldsymbol{\$}}_{w l 2} & \hat{\boldsymbol{\$}}_{w l 3} & \hat{\mathbf{\$}}_{w r 1} & \hat{\boldsymbol{\$}}_{w r 2} & \hat{\boldsymbol{\$}}_{w r 3}
\end{array}\right], \\
& \boldsymbol{k}_{E}=\operatorname{diag}\left(\begin{array}{llllll}
k_{l 1} & k_{l 2} & k_{l 3} & k_{r 1} & k_{r 2} & k_{r 3}
\end{array}\right),
\end{aligned}
$$

where $\boldsymbol{W}_{E}$ is the matrix of eigen-wrenches; $\hat{\boldsymbol{\$}}_{w l i}(i=1,2,3)$ are three eigen-forces in three orthogonal directions and $\boldsymbol{k}_{l i}$ are their linear stiffness; $\hat{\mathbf{\$}}_{w r i}(i=1,2,3)$ are three eigen-moments in three orthogonal directions and $\boldsymbol{k}_{r i}$ are their torsional stiffness; $\boldsymbol{k}_{E}$ is the stiffness characteristics matrix of the CPPPMS. The algorithm to calculate the stiffness characteristics is given in Table I.

It should be noted that the linear stiffness in the $x$ - and $y$-axes is equal to infinity and the linear stiffness in the $z$-axis is equal to $k_{s}$. Therefore, we only concentrate on the three torsional stiffness $\boldsymbol{k}_{r i}(i=1,2,3)$. When one of $\boldsymbol{k}_{r i}$ is approaching to zero, the torsional stiffness in the direction of $\hat{\mathbf{\$}}_{w r i}$ is small and a small extend moment causes an infinite deformation in this direction. That is to say, the motion in the direction of $\hat{\boldsymbol{\$}}_{w r i}$ is uncontrollable, i.e. there is a singularity in this direction. ${ }^{33}$ Therefore, the minimum $\boldsymbol{k}_{r i}$ can be used to identify the stiffness characteristics as well as singularity of the CPPPMS.

\subsection{Dimensions optimization}

Two dimensions of the mechanism are still unknown and will be optimized in this section. The designed variables $v_{1}$ and $v_{2}$ represent the locations of the joints between the cables and the Up-platform. For the optimization of the kinematic design, one may be interested in an index that represents a global property of the mechanism. If we assume that the workspace is $\alpha \in[-\pi / 2, \pi / 2], \quad \beta \in[-\pi / 3, \pi / 3], \quad \gamma \in[-\pi / 3, \pi / 3]$, and $h_{1}=0.4$ (the variable $h 1$ is given as constant, since in real application the change of $h 1$ is small), we call $\bar{k}_{r}$ the global minimum 


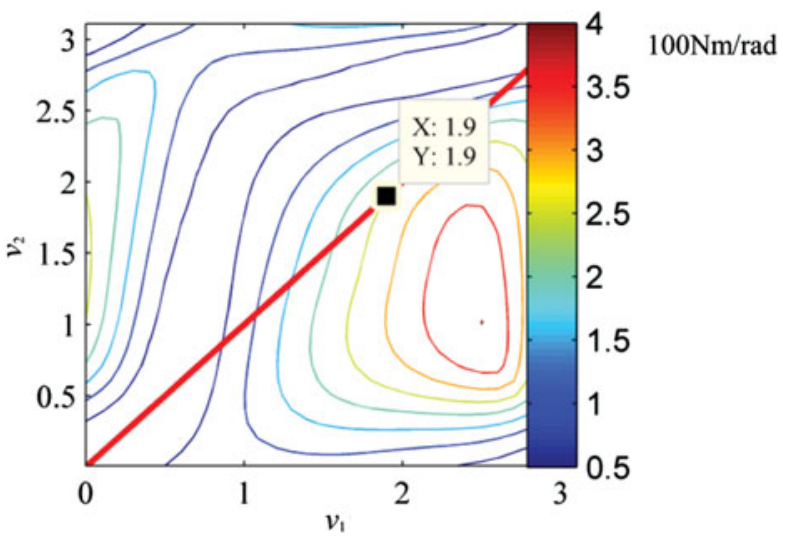

Fig. 4. Variation tendency of $\bar{k}_{r}$ over $v_{1}$ and $v_{2}$, the red line means $v_{1}=v_{2}$.

torsional stiffness, and the definition of $\bar{k}_{r}$ is given as ${ }^{36,37}$

$$
\begin{gathered}
\bar{k}_{r}=\frac{\int_{W} k_{r, \min }\left\|\boldsymbol{J}_{r}\right\| d \boldsymbol{W}}{\int_{W}\left\|\boldsymbol{J}_{r}\right\| d \boldsymbol{W}}=\frac{\int_{-\pi / 3}^{\pi / 3} \int_{-\pi / 3}^{\pi / 3} \int_{-\pi / 2}^{\pi / 2} k_{r, \min }\left\|\boldsymbol{J}_{r}\right\| d \alpha d \beta d \gamma}{\int_{-\pi / 3}^{\pi / 3} \int_{-\pi / 3}^{\pi / 3} \int_{-\pi / 2}^{\pi / 2}\left\|\boldsymbol{J}_{r}\right\| d \alpha d \beta d \gamma} \\
k_{r, \min }=\min \left(\left[k_{r 1}, k_{r 2}, k_{r 3}\right]\right) \quad\left\|\boldsymbol{J}_{r}\right\|=\cos (\beta),
\end{gathered}
$$

in which $k_{r \text {,min }}$ is the minimum torsional stiffness at a particular point of the mechanism workspace $W,\left\|\boldsymbol{J}_{r}\right\|$ is the absolute value of the Jacobian determinant. $\bar{k}_{r}$ is used as an index to optimize the dimensions of the mechanism. If $\bar{k}_{r}$ is large, the global minimum torsional stiffness of the CPPPMS is large, and vice versa. There is a limitation of $v_{1}$ and $v_{2}$, which is $v_{1} \leq v_{2}$. It means that the cables cannot be intersected with each other, because the undersigned dynamics can be caused by the cables intersection. Figure 4 shows the variation tendency of $\bar{k}_{r}$ over $v_{1}$ and $v_{2}$. The red line is the limitation condition which means $v_{1}=v_{2}$. Even though the mean stiffness $\vec{k}_{r}$ reaches the maximum value on the right side of the red line, the optimal values of the dimensions is chosen as $v_{1}=v_{2}=1.9$ to avoid cables intersection. We use these values for afterward analysis.

Once the dimensions are obtained, we can analyze the real workspace and the stiffness map of the CPPPMS. The real workspace is the workspace without singularity. Figure 5 shows the real workspace of $\beta$ and $\gamma$ when $\alpha=0$, and the $k_{r \text {,min }}$ throughout the real workspace. The real workspace is symmetrical with $\gamma$, and the stiffness near the center of the workspace is larger than that near the boundary. The real workspace of $\beta$ and $\gamma$ changes with $\alpha$. Figure 6 shows the real workspace when $\alpha=90^{\circ}$, which is asymmetrical and the peak of the stiffness is shifted to lower right corner of the figure. Figure 7 shows the complete real workspace of $\alpha, \beta$, and $\gamma$ which is axial symmetrical with $\alpha$. As a conclusion, the CPPPMS has high stiffness on its workspace which is larger than $100 \mathrm{Nm} / \mathrm{rad}$. Compared with Figs. 1 and 2, the structure of the CPPPMS is partly similar to the muscle distribution of human neck, and the CPPPMS has similar real workspace with the human neck.

\section{Dynamic Model}

The dynamic model of the CPPPMS consists of three parts, which are the dynamic model of the Up-platform, the dynamic model of the cables, and the dynamic model of the pneumatic muscle active support.

\subsection{The dynamic model of the pneumatic muscle active support}

A pneumatic muscle is made by a rubber tube surrounded by braided shells. When the tube is pressured, the pneumatic muscle is expanded, which leads to the contraction of the pneumatic muscle. ${ }^{22,23}$ The 


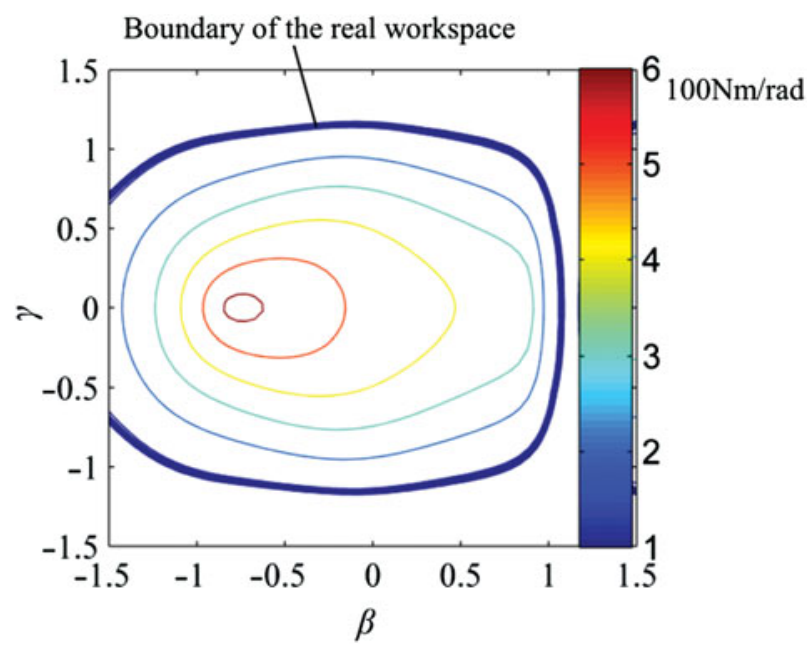

Fig. 5. Minimum $k_{r i}$ throughout the real workspace when $\alpha=0$.

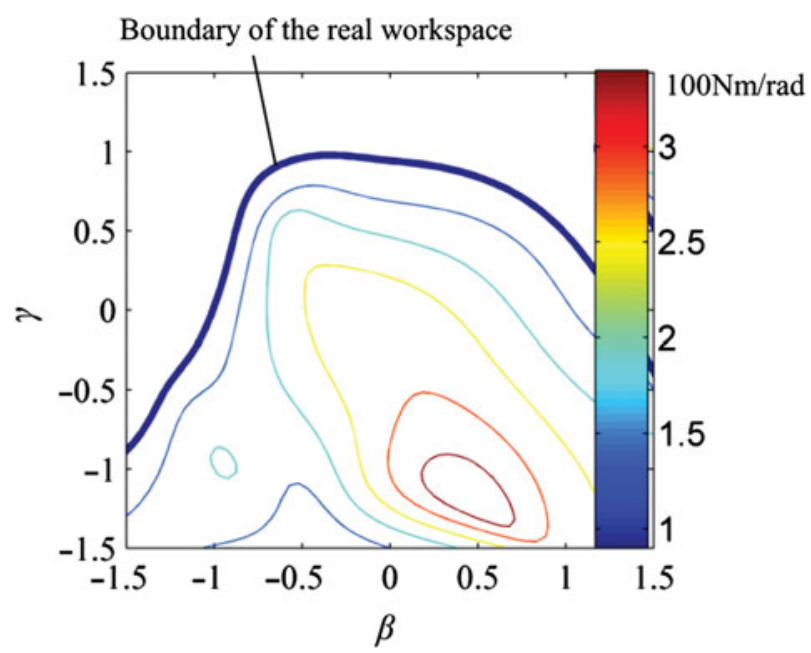

Fig. 6. Minimum $k_{r i}$ throughout the real workspace when $\alpha=90^{\circ}$.

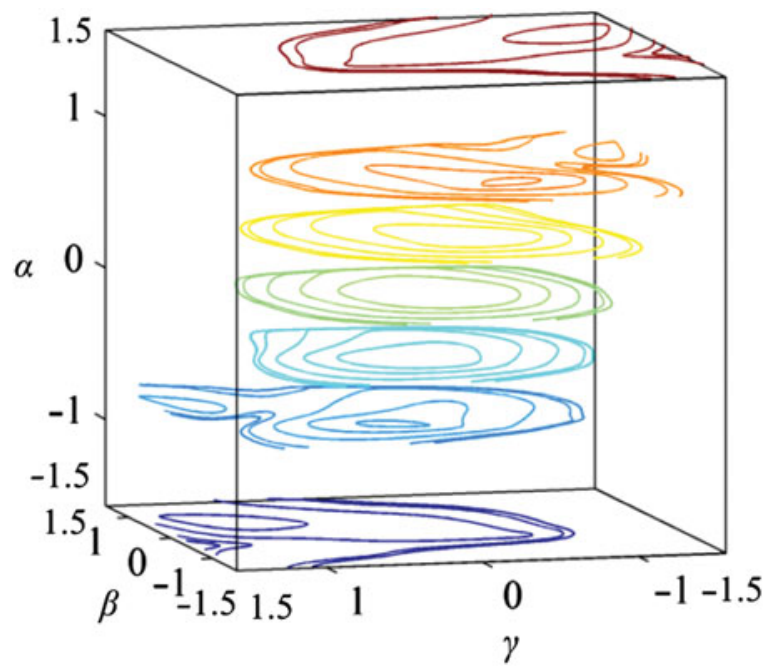

Fig. 7. The complete real workspace of the CPPPMS. 


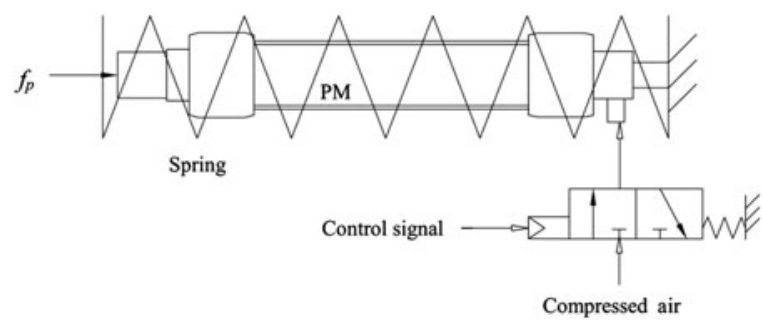

Fig. 8. Structure of the pneumatic muscle active support and the fast switching valve.

force is generated by the inner pressure of the pneumatic muscle. The pressure-force transformation model is first proposed by Chou and Hannaford ${ }^{23}$ with the principle of the virtual work. The input work of the pneumatic muscle is generated by the pressurized gas inside the bladder, and the output work is the external force multiplied by the contraction of the pneumatic muscle,

$$
\begin{aligned}
d W_{I} & =\left(P-P_{0}\right) d V, \\
d W_{O} & =-F d S, \\
F & =-\left(P-P_{0}\right) \frac{d V}{d S},
\end{aligned}
$$

where $P$ is the absolute pressure, and $P_{0}$ is the atmospheric pressure, $F$ is the axial force provided by the pneumatic muscle, $S$ is the length of the pneumatic muscle. To estimate $d V / d S$, it is assumed that the threads in the braided mesh are inextensible. According to, ${ }^{22}$ the volume of the pneumatic muscle is expressed as

$$
\begin{aligned}
V=a S^{3}+b S, \quad a & =-\frac{1}{4 \pi n^{2}}, \quad b=\frac{T_{d}^{2}}{4 \pi n^{2}}, \\
\frac{d V}{d S} & =3 a S^{2}+b,
\end{aligned}
$$

where $n$ is the number of turns of the thread, $T_{d}$ is the thread length. Substituting Eqs. (16) into (14), the pressure-force transformation model is obtained as

$$
F=-\left(3 a S^{2}+b\right)\left(P-P_{0}\right)
$$

In addition, a spring connects to the both sides of the pneumatic muscle, which compose a pneumatic muscle active support shown in Fig. 8. The main function of the active support is absorbing the impact from outside. The force provided by the pneumatic muscle active support is

$$
\begin{aligned}
& f_{p}=-\left(3 a S^{2}+b\right)\left(P-P_{0}\right)+\Delta S k_{s}, \quad \text { if } 3 a S^{2}+b<0, \\
& f_{p}=\Delta S k_{s}, \quad \text { if } 3 a S^{2}+b \geq 0,
\end{aligned}
$$

where $k_{s}$ is the stiffness of the spring. It should be noticed that the pneumatic muscle can only provide tension but not thrust, when the pneumatic muscle is compressed, the force of the pneumatic muscle active support is equal to the force of the spring.

The force of the pneumatic muscle is converted by the inner pressure of the pneumatic muscle, which can be calculated by the gas dynamics. By assuming that the air is ideal gas undergoing an 
adiabatic process

$$
\begin{aligned}
P V & =m_{g} R_{g} T_{g}, \quad P_{\rho}^{-\lambda}=\mathrm{const}, \\
\dot{P} & =\lambda \frac{R_{g} T_{g}}{V} \dot{m}_{g}-\lambda \frac{P}{V} \dot{V}, \\
\dot{V} & =3 a S^{2} \dot{S}+b \dot{S},
\end{aligned}
$$

where $\rho$ is the density of air; $\lambda$ is the ratio of specific heats; $R_{g}$ is the gas constant; $T_{g}$ is the thermodynamic temperature inside the pneumatic muscle; $V$ is the pneumatic muscle's inner volume as a function of $S ; m_{g}$ is the mass of air; $\dot{m}_{g}$ is the mass flow.

Substituting Eqs. (15) and (22) into (21), the pressure change rate can be expressed as

$$
\begin{aligned}
\dot{P} & =\lambda \frac{R_{g} T_{g}}{a S^{3}+b S} \dot{m}_{g}-\lambda \frac{\left(3 a S^{2}+b\right) \dot{S}}{a S^{3}+b S} P, \\
\dot{m}_{g} & =K_{m}(P, \operatorname{sign}(\dot{P})) U_{P}, \\
K_{m}(P, \operatorname{sign}(\dot{P})) & = \begin{cases}A_{e}\left(P_{u} / \sqrt{R T}\right) \sqrt{\lambda\left(\frac{2}{\lambda+1}\right)^{\frac{\lambda+1}{\lambda-1}}} & \text { if } \frac{P_{d}}{P_{u}}<0.528 \\
A_{e}\left(P_{u} / \sqrt{R T}\right) \sqrt{\frac{2 \lambda}{\lambda-1}\left[\left(\frac{P d}{P u}\right)^{\frac{2}{\lambda}}-\left(\frac{P d}{P u}\right)^{\frac{\lambda+1}{\lambda}}\right]} & \text { if } \frac{P_{d}}{P_{u}} \geq 0.528\end{cases}
\end{aligned}
$$

where $\dot{m}_{g}$ is controlled by a fast switching valve, the input value of the fast switching valve is the voltage $U_{P}, K_{m}(P, \operatorname{sign}(\dot{P}))$ is a nonlinear flow gain function given by, ${ }^{25} A_{e}$ is the effective orifice area of the fast switching valve, $P_{u}$ is the upstream pressure, and $P_{d}$ is the downstream pressure.

Therefore, the dynamics of the pneumatic muscle active support can be written as

$$
\begin{gathered}
\left\{\begin{array}{l}
f_{p}=-\left(3 a S^{2}+b\right)\left(P-P_{0}\right)+\Delta S k_{s}, \quad \text { if } 3 a S^{2}+b<0 \\
f_{p}=\Delta S k_{s}, \quad \text { if } 3 a S^{2}+b \geq 0
\end{array},\right. \\
\dot{P}=\lambda \frac{R_{g} T_{g}}{a S^{3}+b S} \dot{m}_{g}-\lambda \frac{\left(3 a S^{2}+b\right) \dot{S}}{a S^{3}+b S} P \\
\dot{m}_{g}=K_{m}(P, \operatorname{sign}(\dot{P})) U_{P},
\end{gathered}
$$

4.2. The dynamic model of the cables

The elastic cable modeling is shown in Fig. 9. ${ }^{19-21}$ The tension within the cable $T$ acting along the cable axial direction is

$$
T=k_{a} \Delta S, \quad k_{a}=\frac{E A_{a}}{S}, \quad \Delta S=R \theta-S,
$$

where $k_{a}$ is the stiffness of the cable; $\Delta S$ is the stretched length; $S$ is the unstretched length; $A_{a}$ is the cross section area of the cable; $E$ is its effective Young's modulus; $\theta$ is the rotary angle of the pulley; and $R$ is its radius.

The friction is along the axes of the cables

$$
F_{d}=\mu(R \dot{\theta}-\dot{S})
$$

where $\mu$ is the damping coefficient of the cable. In order to insure certain tension, $\dot{\theta}$ cannot be too big due to the limited power of the servo motor. Therefore, we assume that $R \dot{\theta} \ll \dot{S}$ and Eq. (28) can be simplified to

$$
F_{d} \approx \mu \dot{S}
$$




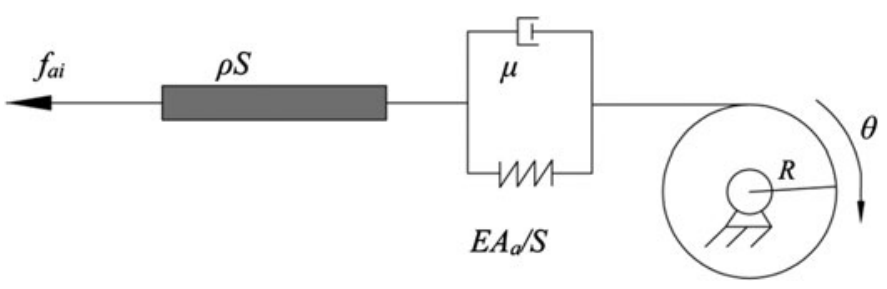

Fig. 9. Schematic of elastic cable modeling.

Compared with other parts of the CPPPMS, the mass of the cables is negligible. Therefore, the gravity and the acceleration force of the cables can be ignored. The dynamic equation of the cable can be written as

$$
\begin{aligned}
& f_{a}=-\mu \dot{S}+\frac{E A_{a}}{S}(R \theta-S), \quad \text { if } R \theta-S>0, \\
& f_{a}=0, \quad \text { if } R \theta-S \leq 0
\end{aligned}
$$

where the cable can only provide tension but not thrust. When the cable is compressed, it cannot provide force.

\subsection{The dynamic model of the Up-platform}

The dynamic model of the Up-platform is solved by Newton-Euler method. The center of mass of the Up-platform is assumed to be located at point $E$, which can be represented as

$$
\boldsymbol{r}_{O E}=\left[\begin{array}{c}
h_{2}(C \alpha S \beta C \gamma+S \alpha S \gamma) \\
h_{2}(S \alpha S \beta C \gamma-C \alpha S \gamma) \\
h_{1}+h_{2} C \beta C \gamma
\end{array}\right],
$$

where $h_{2}$ is the distance between the point $C$ and $E$. The velocity of the center of mass is

$$
\dot{\boldsymbol{r}}_{O E}=\boldsymbol{J}_{t} \dot{\boldsymbol{q}}
$$

where $\boldsymbol{q}=\left[\alpha, \beta, \gamma, h_{1}\right]$, which is the state variable of the system, and $\dot{\boldsymbol{q}}$ is the time derivative of $\boldsymbol{q}$. The acceleration of the center of mass is

$$
\ddot{\boldsymbol{r}}_{O E}=\boldsymbol{J}_{t} \ddot{\boldsymbol{q}}+\dot{\boldsymbol{J}}_{t} \dot{\boldsymbol{q}}
$$

where $\dot{\boldsymbol{J}}_{t}$ is the time derivative of $\boldsymbol{J}_{t}$. The angle velocity of the Up-platform is

$$
\dot{\delta}=J_{r} \dot{\boldsymbol{q}}
$$

The angle acceleration of the Up-platform is

$$
\ddot{\boldsymbol{\delta}}=\boldsymbol{J}_{r} \ddot{\boldsymbol{q}}+\dot{\boldsymbol{J}}_{r} \dot{\boldsymbol{q}},
$$

where $\dot{\boldsymbol{J}}_{r}$ is the time derivative of $\boldsymbol{J}_{r}$. Using Newton-Euler method based on virtual work principle, the dynamics of the Up-platform is

$$
\boldsymbol{J}_{t}^{\mathrm{T}}\left(m_{u} \boldsymbol{J}_{t} \ddot{\boldsymbol{q}}+m_{u} \dot{\boldsymbol{J}}_{t} \dot{\boldsymbol{q}}\right)+\boldsymbol{J}_{r}^{\mathrm{T}}\left(\boldsymbol{I}_{u} \boldsymbol{J}_{r} \ddot{\boldsymbol{q}}+\boldsymbol{I}_{u} \dot{\boldsymbol{J}}_{r} \dot{\boldsymbol{q}}\right)=\boldsymbol{J}_{t}^{\mathrm{T}}\left(\boldsymbol{G}+\boldsymbol{F}_{s}\right)+\boldsymbol{J}_{r}^{\mathrm{T}} \boldsymbol{M}_{s},
$$

where $m_{u}$ is the mass of the Up-platform; $\boldsymbol{I}_{u}$ is moment of inertia of the Up-platform respected to the coordinate $K_{E} ; \boldsymbol{F}_{s}$ and $\boldsymbol{M}_{s}$ are the external force and the moment imposed on the Up-platform, respectively; $\boldsymbol{G}$ is the vector represented as gravity. Reforming the Eq. (37), the dynamics of the 
Up-platform is

$$
\boldsymbol{J} \ddot{\boldsymbol{q}}+\boldsymbol{\eta}=\boldsymbol{H}\left[\begin{array}{llllll}
f_{a 1} & f_{a 2} & f_{a 3} & f_{a 4} & f_{a 5} & f_{p}
\end{array}\right]^{\mathrm{T}},
$$

where $f_{p}$ is the force generated by the pneumatic muscle active support, which is analyzed in Section 4.1; $f_{a i}(i=1,2,3,4,5)$ are tensions generated by the cables, which are given in Section 4.2; $\boldsymbol{J}, \boldsymbol{\eta}$ and $\boldsymbol{H}$ are given in Appendix A.

\subsection{The dynamic model of the CPPPMS}

Based on aforementioned analysis, the whole dynamic model consists of the pneumatic muscle active support model, the cables model, and the Up-platform model. The unstretched length of the $i$ th cable in the CPPPMS is approximately equal to $S_{A B i}$; the length of the pneumatic muscle active support is $h_{1}$; the unstretched length of the spring is $h_{10}$. Then the dynamics of the CPPPMS is expressed as

$$
\begin{gathered}
\boldsymbol{J} \ddot{q}+\boldsymbol{\eta}+\boldsymbol{\eta}_{f}=\boldsymbol{H}_{f} \boldsymbol{\tau}, \\
\boldsymbol{\tau}=\left[\theta_{a 1}, \theta_{a 2}, \theta_{a 3}, \theta_{a 4}, \theta_{a 5}, P\right]^{T}, \\
\dot{P}=\lambda \frac{R_{g} T_{g}}{a S^{3}+b S} K_{m}(P, \operatorname{sign}(\dot{P})) U_{p}-\lambda \frac{\left(3 a S^{2}+b\right) \dot{S}}{a S^{3}+b S} P,
\end{gathered}
$$

where $\mathbf{J}$ and $\boldsymbol{\eta}$ are given in Appendix A; $\boldsymbol{\eta}_{f}$ and $\boldsymbol{H}_{f}$ are the flexible terms, given in Appendix B. One can see that there are flexible terms in the dynamics due to the flexible actuators. Due to the flexible terms, the solution of the dynamic equation may not exit for some conditions. (If one or more cables are compressed, the system will lose one or more actuators.) Therefore, redundant actuators are required to ensure that the dynamic equation has at least one solution in the designed workspace. Moreover, the redundant actuators make the inverse dynamic equation of the CPPPMS to be an underdetermined equation, and an optimized method is proposed later to obtain the best driven strategy.

\section{Control for the CPPPMS}

In this section, a sliding mode control is proposed for trajectory tracking of the CPPPMS, which is a redundant driven system with the flexibility and uncertainties of the dynamics. The stability of the control system is verified by a Lyapunov function. In addition, a PD control is presented for a comparative study.

\subsection{Sliding mode control for the flexible dynamic model}

Modeling uncertainties and unmodeled dynamics exist in the CPPPMS system. For example, there are obvious hysteresis phenomena in the dynamic feature of the pneumatic muscle, ${ }^{27}$ which seems impossible to be described accurately by the mathematic method. The mass and the gravity of the cables are ignored in the dynamic modeling, which results in the uncertainties of the dynamic model. The uncertain terms of the CPPPMS are expressed as

$$
\begin{aligned}
\boldsymbol{J}(\boldsymbol{q}) & =\boldsymbol{J}_{0}(\boldsymbol{q})+\Delta J(\boldsymbol{q}) \\
\eta(\boldsymbol{q}, \dot{\boldsymbol{q}}) & =\boldsymbol{\eta}_{0}(\boldsymbol{q}, \dot{\boldsymbol{q}})+\Delta \eta(\boldsymbol{q}, \dot{\boldsymbol{q}}),
\end{aligned}
$$

where $\boldsymbol{J}_{0}(\boldsymbol{q})$ and $\boldsymbol{\eta}_{0}(\boldsymbol{q}, \dot{\boldsymbol{q}})$ are estimated terms, which are given in Appendix A, and $\boldsymbol{\Delta} \boldsymbol{J}(\boldsymbol{q})$ and $\Delta \boldsymbol{\eta}(\boldsymbol{q}, \dot{\boldsymbol{q}})$ are uncertain terms, which are unknown but bounded. $\boldsymbol{\Delta} \boldsymbol{J}(\boldsymbol{q})$ includes the ignored mass of the cables and the pneumatic muscle, and $\Delta \boldsymbol{\eta}(\boldsymbol{q}, \dot{\boldsymbol{q}})$ includes the error of damping of the system, the hysteresis phenomena of the pneumatic muscle, and the ignored gravity of the cables. Then, the dynamic equation of the CPPPMS is written in the following form

$$
\begin{aligned}
& \boldsymbol{J}_{0}(\boldsymbol{q}) \ddot{\boldsymbol{q}}+\boldsymbol{\eta}_{0}(\boldsymbol{q}, \dot{\boldsymbol{q}})+\boldsymbol{\eta}_{f}(\boldsymbol{q})=\boldsymbol{H}_{f} \boldsymbol{\tau}+\boldsymbol{\rho} \\
& \boldsymbol{\rho}=-\Delta J(\boldsymbol{q}) \ddot{\boldsymbol{q}}-\boldsymbol{\Delta} \eta(\boldsymbol{q}, \dot{\boldsymbol{q}}) .
\end{aligned}
$$


Compared with the whole system, the uncertain terms are small and limited in a certain range. Therefore, it is reasonable to make following assumptions

$$
\|\boldsymbol{\rho}\|<n_{b 0}+n_{b 1}\|\boldsymbol{q}\|+n_{b 2}\|\dot{\boldsymbol{q}}\|^{2},
$$

where $n_{b 0}, n_{b 1}, n_{b 2}$ are positive constants, the values of them can be identified by experiment.

Theorem 1. It is supposing that $\boldsymbol{q}_{r}$ is the desired input of the CPPPMS and $\dot{\boldsymbol{q}}_{r}$ is the derivative of $\boldsymbol{q}_{r}$, then the error is $\boldsymbol{\varepsilon}=\boldsymbol{q}-\boldsymbol{q}_{r}$, and $\dot{\boldsymbol{\varepsilon}}$ is the derivative of $\boldsymbol{\varepsilon}$. For the CPPPMS, if the manifold of the sliding mode control is chosen as

$$
\boldsymbol{s}=\boldsymbol{\varepsilon}+\boldsymbol{C}_{1} \dot{\boldsymbol{\varepsilon}}^{p},
$$

where $\boldsymbol{C}_{1}=\operatorname{diag}\left[C_{11}, \ldots, C_{14}\right]$ is a design matrix, and $p$ is a control parameter, and the control input $\tau$ satisfies the following relationship

$$
\boldsymbol{H}_{f} \boldsymbol{\tau}=\boldsymbol{\tau}_{0}+\boldsymbol{u}_{0}+\boldsymbol{u}_{1},
$$

here

$$
\begin{aligned}
\boldsymbol{\tau}_{0}= & \boldsymbol{\eta}_{0}(\boldsymbol{q}, \dot{\boldsymbol{q}})+\boldsymbol{\eta}_{f}(\boldsymbol{q})+\boldsymbol{J}_{0}(\boldsymbol{q}) \ddot{\boldsymbol{q}}, \\
\boldsymbol{u}_{0}= & -\frac{1}{p} \boldsymbol{J}_{0}(\boldsymbol{q}) \boldsymbol{C}_{1}^{-1} \dot{\boldsymbol{\varepsilon}}^{2-p}, \\
\boldsymbol{u}_{1}= & -\frac{1}{p} \frac{\left[\boldsymbol{s}^{\mathrm{T}} \boldsymbol{C}_{1} \operatorname{diag}\left(\dot{\boldsymbol{\varepsilon}}^{p-1}\right) \boldsymbol{J}_{0}^{-1}(\boldsymbol{q})\right]^{\mathrm{T}}}{\left\|\boldsymbol{s}^{\mathrm{T}} \boldsymbol{C}_{1} \operatorname{diag}\left(\dot{\boldsymbol{\varepsilon}}^{p-1}\right) \boldsymbol{J}_{0}^{-1}(\boldsymbol{q})\right\|} \\
& \times\left[\|\boldsymbol{s}\|\left\|\boldsymbol{s}^{\mathrm{T}} \mathbf{C}_{1} \operatorname{diag}\left(\dot{\boldsymbol{\varepsilon}}^{p-1}\right) \boldsymbol{J}_{0}^{-1}(\boldsymbol{q})\right\|\left(n_{b 0}+n_{b 1}\|\boldsymbol{q}\|+n_{b 2}\|\dot{\boldsymbol{q}}\|^{2}\right)\right],
\end{aligned}
$$

the system tracking error $\boldsymbol{\varepsilon}(t)$ will be converged to zero for infinite time. ${ }^{28,29}$

Equation (44) is an underdetermined equation, which has an infinitude of solutions. In order to obtain an unique solution, a performance index has to be optimized and be used as a constraint. ${ }^{38}$ The conditions of optimization are given as follows:

1. The tension of the cables has to be stronger than the minimum force $f_{a, \min }$ to ensure that the cables are tightened; meanwhile, the tension has to be weaker than the maximum force $f_{a \text {,max }}$ which is the maximum force generated by the servo motor;

2. The force of the cables should be close to $\bar{f}_{a}$, which is the mean force between $f_{a, \min }$ and $f_{a, \max }$. The advantage of using the mean force as the objective is that it has the same variation range of increase and decrease in force;

3. The pressure of the pneumatic muscle has to be higher than the ambient pressure as $1 \mathrm{e} 5 \mathrm{~Pa}$, and lower than $5 \mathrm{e} 5 \mathrm{~Pa}$ which is the maximum pressure of pneumatic muscle allowed; ${ }^{22}$

4. The pressure of the pneumatic muscle should be close to $3 \mathrm{e} 5 \mathrm{~Pa}$, which is the mean pressure between the minimum and maximum amount of pressure.

According to the above conditions, the performance index is given as

$$
\min \sum_{i}^{N=6} \sigma_{i}, \quad \sigma_{i}=\left\|\left(R \theta_{i}-S_{A B i}\right) \frac{E A_{a}}{S_{A B i}}-\bar{f}_{a i}\right\|, \quad(i=1,2,3,4,5) \text { and } \sigma_{6}=\|P-3 e 5\|,
$$

subject to

$$
\boldsymbol{H}_{f} \boldsymbol{\tau}=\boldsymbol{\tau}_{0}+\boldsymbol{u}_{0}+\boldsymbol{u}_{1}
$$


and

$$
\begin{aligned}
\left(\frac{f_{a, \min } S_{A B i}}{E A_{a}}+S_{A B i}\right) / R \leq \theta_{i} \leq\left(\frac{f_{a, \max } S_{A B i}}{E A_{a}}+S_{A B i}\right) / R, & (i=1,2,3,4,5), \\
& \text { and } 1 \mathrm{e} 5 \leq P \leq 5 \mathrm{e} 5,
\end{aligned}
$$

where $\sigma_{i}$ is the performance index. Mathematically, this problem is known as quadratic programming. Efficient iterative algorithms for the resolution of quadratic programs can be easily found in general optimization packages of Matlab.

Proof. The Theorem 1 can be proved by Lyapunov theory. By considering the following Lyapunov function,

$$
\boldsymbol{L}=\frac{1}{2} \boldsymbol{s}^{\mathrm{T}} \boldsymbol{s} .
$$

We assume that Eq. (44) has a valid solution. By differentiating $\mathbf{L}$ with time and substituting Eqs. (42)-(47) into $\dot{\boldsymbol{L}}$, yields,

$$
\begin{aligned}
\dot{\boldsymbol{L}}= & \boldsymbol{s}^{\mathrm{T}} \dot{\boldsymbol{s}}=\boldsymbol{s}^{\mathrm{T}}\left(\dot{\boldsymbol{\varepsilon}}+p \mathbf{C}_{1} \operatorname{diag}\left(\boldsymbol{\varepsilon}^{p-1}\right) \ddot{\boldsymbol{\varepsilon}}\right) \\
= & \boldsymbol{s}^{\mathrm{T}}\left(\dot{\boldsymbol{\varepsilon}}+p \boldsymbol{C}_{1} \operatorname{diag}\left(\boldsymbol{\varepsilon}^{p-1}\right) \boldsymbol{J}_{0}^{-1}(\boldsymbol{q})\left(\boldsymbol{J}_{0}(\boldsymbol{q}) \ddot{\boldsymbol{q}}_{r}-\boldsymbol{\eta}(\boldsymbol{q}, \dot{\boldsymbol{q}})-\boldsymbol{\eta}_{f}(\boldsymbol{q})+\boldsymbol{\rho}+\boldsymbol{\tau}_{0}+\boldsymbol{u}_{0}+\boldsymbol{u}_{1}\right)\right) \\
= & -p\|\boldsymbol{s}\|\left\|\boldsymbol{C}_{1} \operatorname{diag}\left(\boldsymbol{\varepsilon}^{p-1}\right) \boldsymbol{J}_{0}^{-1}(\boldsymbol{q})\right\| \times\left(n_{b 0}+n_{b 1}\|\boldsymbol{q}\|+n_{b 2}\|\dot{\boldsymbol{q}}\|^{2}\right)+p \boldsymbol{s}^{\mathrm{T}} \boldsymbol{C}_{1} \operatorname{diag}\left(\boldsymbol{\varepsilon}^{p-1}\right) \boldsymbol{J}_{0}^{-1}(\boldsymbol{q}) \boldsymbol{\rho} \\
& \quad-p\|\boldsymbol{s}\|\left\|\boldsymbol{C}_{1} \operatorname{diag}\left(\boldsymbol{\varepsilon}^{p-1}\right) \boldsymbol{J}_{0}^{-1}(\boldsymbol{q})\right\| \times\left(n_{b 0}+n_{b 1}\|\boldsymbol{q}\|+n_{b 2}\|\dot{\boldsymbol{q}}\|^{2}\right) \\
& \quad+p\|\boldsymbol{s}\|\left\|\boldsymbol{C}_{1} \operatorname{diag}\left(\boldsymbol{\varepsilon}^{p-1}\right) \boldsymbol{J}_{0}^{-1}(\boldsymbol{q})\right\|\|\boldsymbol{\rho}\| \\
= & -p\|\boldsymbol{s}\|\left\|\boldsymbol{C}_{1} \operatorname{diag}\left(\boldsymbol{\varepsilon}^{p-1}\right) \boldsymbol{J}_{0}^{-1}(\boldsymbol{q})\right\| \times\left(n_{b 0}+n_{b 1}\|\boldsymbol{q}\|+n_{b 2}\|\dot{\boldsymbol{q}}\|^{2}-\|\boldsymbol{\rho}\|\right)\|\boldsymbol{s}\|
\end{aligned}
$$

That is

$$
\dot{\boldsymbol{L}} \leq-\boldsymbol{Q}\|\boldsymbol{s}\|<0
$$

where

$$
\boldsymbol{Q}=p\|\boldsymbol{s}\|\left\|\boldsymbol{C}_{1} \operatorname{diag}\left(\boldsymbol{\varepsilon}^{p-1}\right) \boldsymbol{J}_{0}^{-1}(\boldsymbol{q})\right\| \times\left({ }_{b 0}+n_{b 1}\|\boldsymbol{q}\|+{ }_{b 2}\|\dot{\boldsymbol{q}}\|^{2}-\|\boldsymbol{\rho}\|\right)>0 .
$$

Therefore, according to Lyapunov stability criterion, ${ }^{29}$ the manifold $s$ in (43) converges to zero in finite time, and the error converges to zero along $s=0$ in finite time.

5.2. P-control for the inner pressure of the pneumatic muscle

The pressure control can be realized by a $P$-control. The input voltage of the fast switching valve is given as

$$
U_{P}=\left\{\begin{array}{ll}
k_{1}\left(P_{r}-P\right), & \text { if } P_{r}-P \leq 0 \\
k_{2}\left(P_{r}-P\right), & \text { if } P_{r}-P>0
\end{array},\right.
$$

where $k_{1}$ and $k_{2}$ are the $P$-gain; $P_{r}$ is the required pressure calculated with Eq. (44), and $P$ is the measured pressure. Combining the $P$-control and the sliding mode control, the posture control of the CPPPMS is given in Fig. 10. In the CPPPMS, the cables are driven by the servo motors, which can output the rotary angle of the pulley. The pressure is controlled by a fast switching valve. Therefore, the control variables are the five rotary angles of the pulleys and the voltage of the fast switching valve. The state variables are the posture of the CPPPMS. 
Table II. System parameters of the CPPPMS and the control parameters.

\begin{tabular}{lccc}
\hline System parameters & Value & Control parameters & Value \\
\hline$m_{u}$ & $2 \mathrm{~kg}$ & $p$ & $25 / 23$ \\
$h_{2}$ & $0.1 \mathrm{~m}$ & $C_{1}$ & 1.2 \\
$R_{a}$ & $0.2 \mathrm{~m}$ & $C_{2}$ & 1.2 \\
$R_{b}$ & $0.2 \mathrm{~m}$ & $C_{3}$ & 1.2 \\
$E$ & $1 \mathrm{GPa}$ & $C_{4}$ & 1.2 \\
$A_{a}$ & $10^{-5} \mathrm{~m}^{2}$ & $k_{1}$ & $2 \mathrm{e}^{-4}$ \\
$\mu$ & 0.1 & $k_{2}$ & $1.5 \mathrm{e}^{-4}$ \\
$a$ & -0.003 & $n_{b 1}$ & 150 \\
$b$ & $0.00075 \mathrm{~m}^{2}$ & $n_{b 2}$ & 1 \\
$k_{s}$ & $10 \mathrm{~N} / \mathrm{mm}$ & $n_{b 3}$ & 1 \\
\hline
\end{tabular}

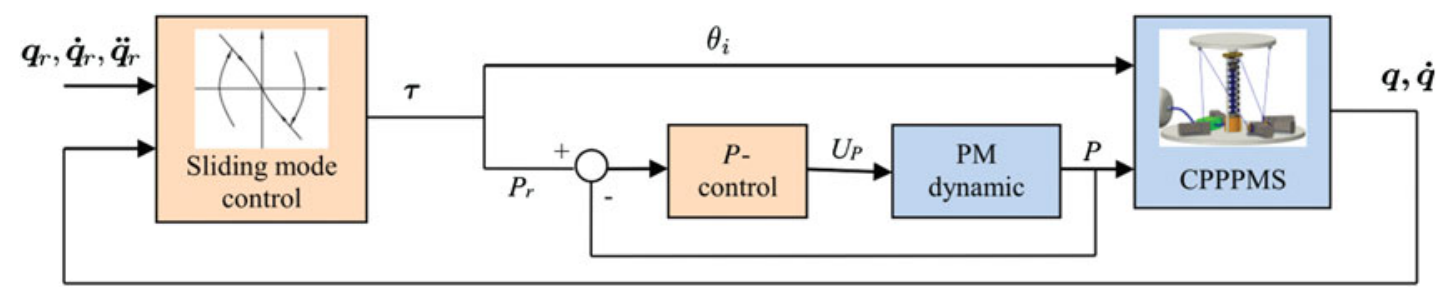

Fig. 10. Schematic diagram of the posture controller using a sliding mode control.

\subsection{Comparison study}

A PD control is proposed for the comparison study. The sliding mode control is replaced with the PD control. The control input of the PD control is given as

$$
\boldsymbol{H}_{f} \boldsymbol{\tau}=\boldsymbol{\tau}_{0}+\boldsymbol{K}_{p} \boldsymbol{\varepsilon}+\boldsymbol{K}_{d} \dot{\boldsymbol{\varepsilon}}
$$

where $\tau_{0}$ is given in Eq. (45), $\boldsymbol{K}_{p}$ and $\boldsymbol{K}_{d}$ are the positive definite gain matrices. In the simulation study, the gain matrices were selected as $\boldsymbol{K}_{p}=20 \boldsymbol{I}$ and $\boldsymbol{K}_{d}=20 \boldsymbol{I}$, with $\mathbf{I}$ as a identity matrix, respectively. The stability of a PD control can be proved in refs. [39, 40]. Equation (53) is an underdetermined equation, its unique solution can be obtained by using the performance index $\sigma_{i}$ as a constraint.

\section{Numerical Results and Discussion}

In this section, the simulation investigation is carried out to detect the control performances of the CPPPMS. All simulations are performed with MATLAB 2010. The simulated time is $10 \mathrm{~s}$ with the step size of $0.01 \mathrm{~s}$. The parameters of the CPPPMS are given in Table II.

The designed trajectory is given as a typical motion deriving from a neck exercise, which is the head moving along a circle. The function of the trajectory is,

$$
\alpha=0, \beta=\pi / 6 \sin (\pi / 5 t), \gamma=\pi / 6 \cos (\pi / 5 t), h_{1}=0.4 .
$$

The uncertainties of the model are assumed as

$$
\Delta J(q)=\operatorname{rand} \times 0.05 \times \boldsymbol{J}_{0}(\boldsymbol{q}), \boldsymbol{\Delta} \eta(q)=\operatorname{rand} \times 0.05 \times \boldsymbol{\eta}_{0}(\boldsymbol{q}),
$$

where "rand" is a pseudorandom scalar drawn from the distribution of open interval $(0,1)$. Two control methods in terms of a sliding mode control and a PD control are used to impose the CPPPMS moving along a designed trajectory.

The simulation results of both controls are given as follows: The trajectory tracking as the endeffector of the Up-platform is given in Fig. 11, and the simulation trajectory is closer to the designed trajectory for the sliding mode controller than the PD controller. The angle error and the angle velocity error for both controllers are proposed in Figs. 12 and 13, while the length and the velocity error 


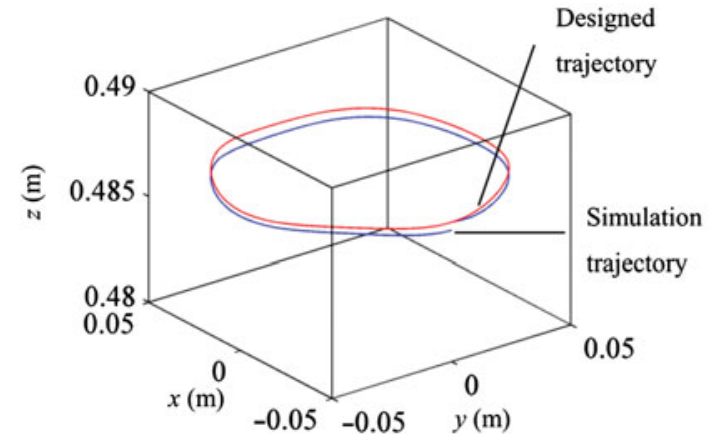

(a)

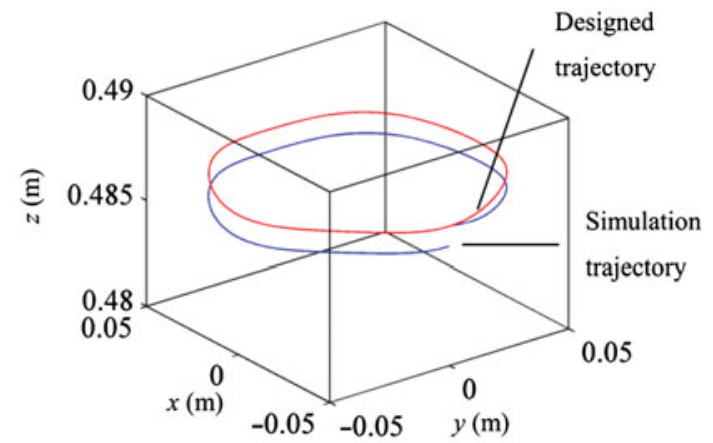

(b)

Fig. 11. Trajectory tracking of the end effector. (a) Sliding mode control. (b) PD control.

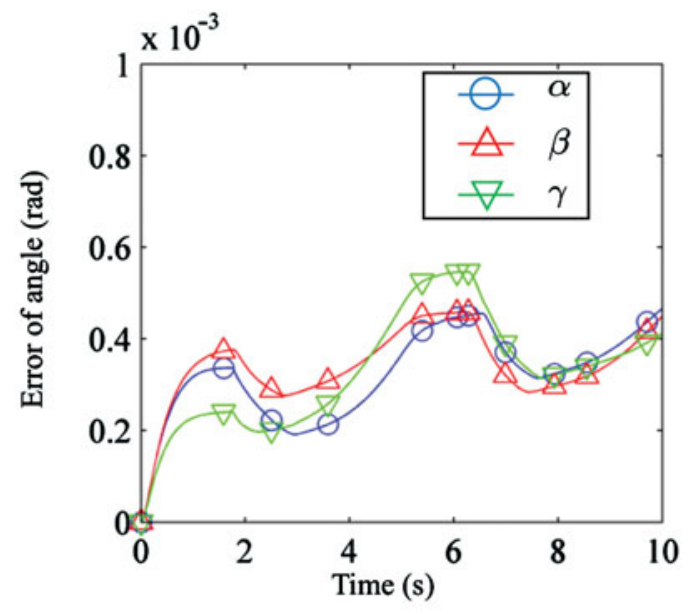

(a)

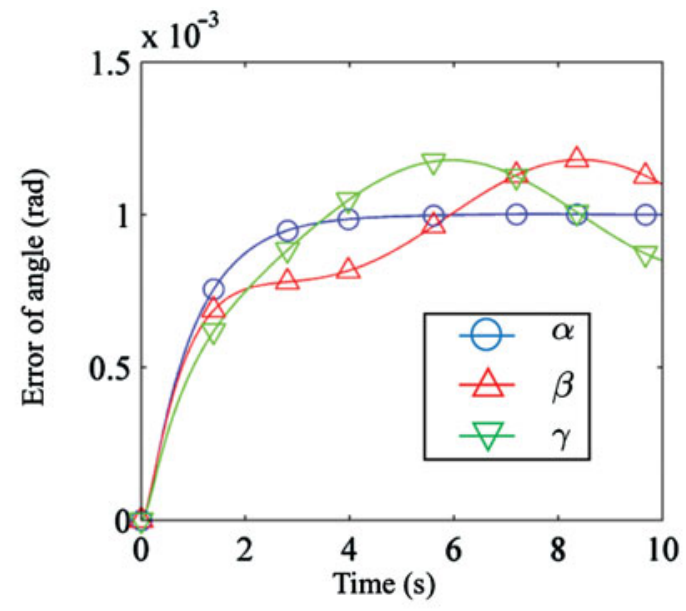

(b)

Fig. 12. Error of the angle $\alpha, \beta, \gamma$. (a) Sliding mode control. (b) PD control.

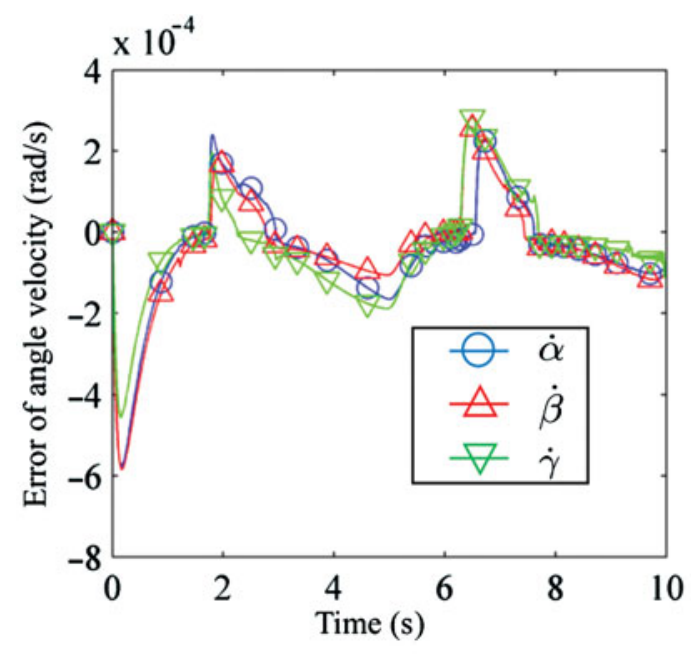

(a)

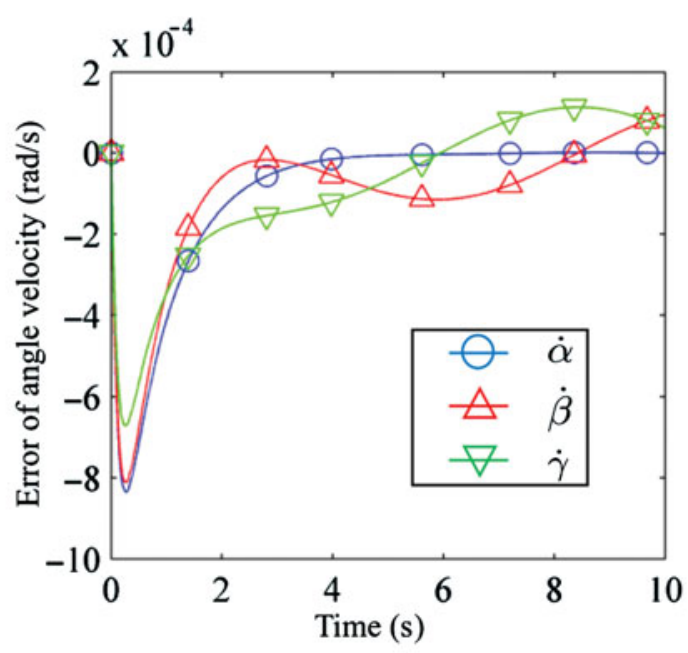

(b)

Fig. 13. Error of angle velocity $\dot{\alpha}, \dot{\beta}, \dot{\gamma}$. (a) Sliding mode control. (b) PD control. 


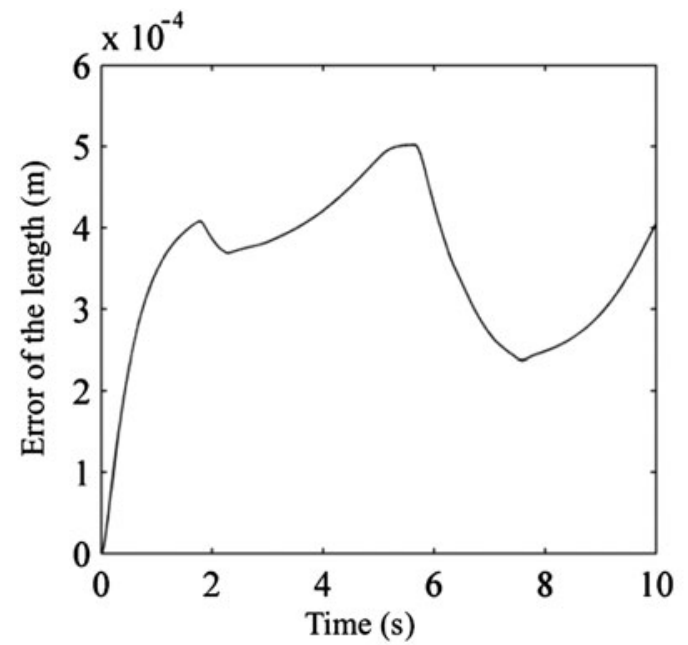

(a)

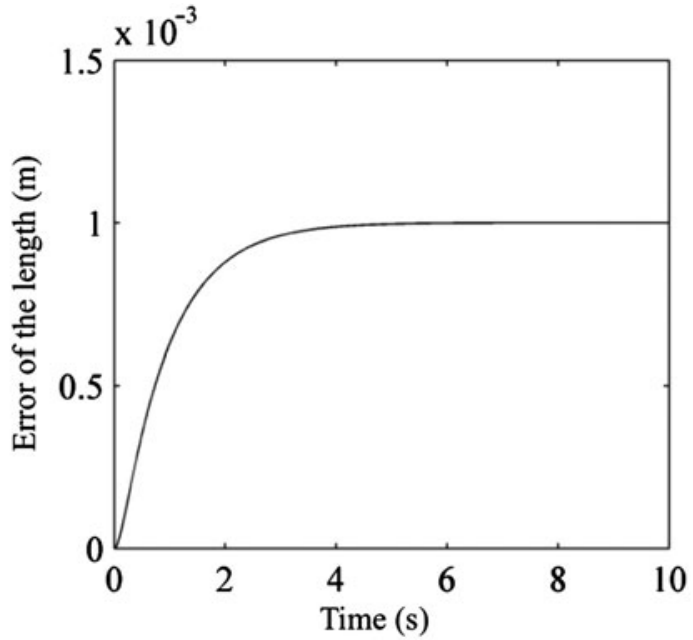

(b)

Fig. 14. Error of the length of the pneumatic muscle active support. (a) Sliding mode control. (b) PD control.

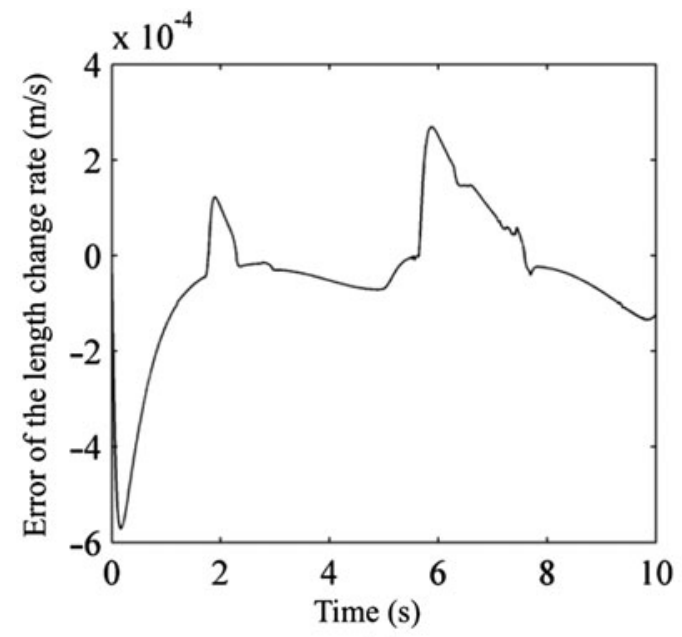

(a)

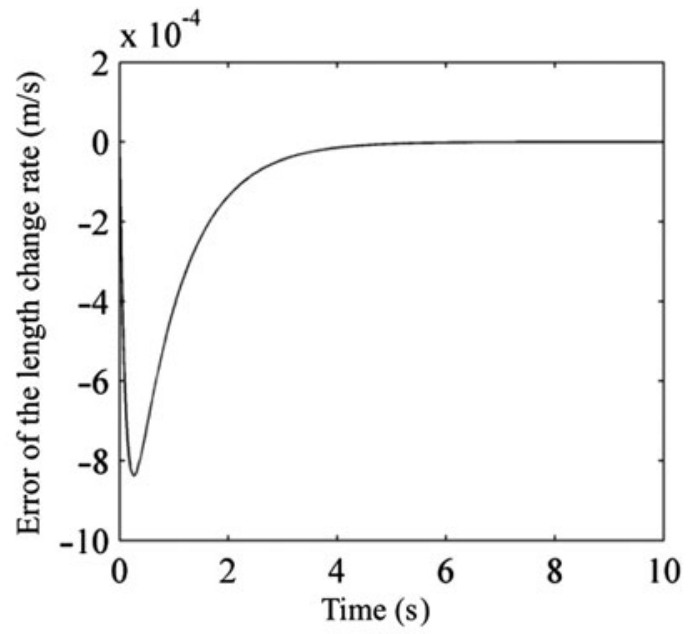

(b)

Fig. 15. Error of the length change rate of the pneumatic muscle active support. (a) Sliding mode control. (b) PD control.

are shown in Figs. 14 and 15. For the angle control and length control, the slide mode controller has a better overall performance than that of the PD controller. However, for the velocity control, the errors in the sliding mode controller have two jumps in $2 \mathrm{~s}$ and $6.5 \mathrm{~s}$ shown in Figs. 13a and 15a, and the errors are larger than that in the PD controller at that moment. The working principle of the slide mode control is that it converts the dynamic behavior of the system to the dynamics of the designed manifold and overcomes the model uncertainty of the system. The jumps appear when the state variables go across the sliding manifold and the control input $u_{1}$ changes its sign. In contrast, the velocity error of the PD control is smooth, but the model uncertainty of the CPPPMS makes it difficult to track the designed trajectory precisely.

Figures 16-18 show the input angle of $i$ th pulley, the absolute pressure in the pneumatic muscle, and the input voltages for both controllers, respectively. Figure 19 shows the poses of the CPPPMS at different instants when it is controlled by the sliding mode controller. One can see that the CPPPMS has the ability to finish the typical motion of human necks.

Table III shows the comparison between both controllers in the sense of the control performance. The maximum angle error for the sliding mode control is $54.17 \%$ smaller than the PD control, and the 


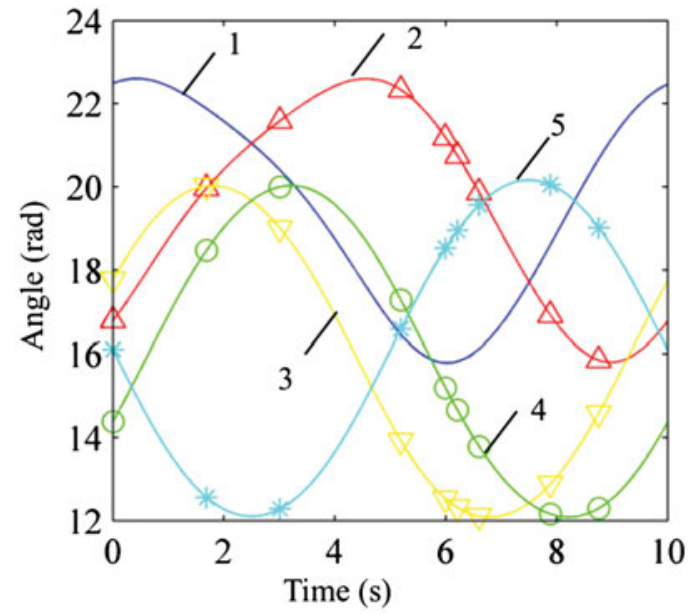

(a)

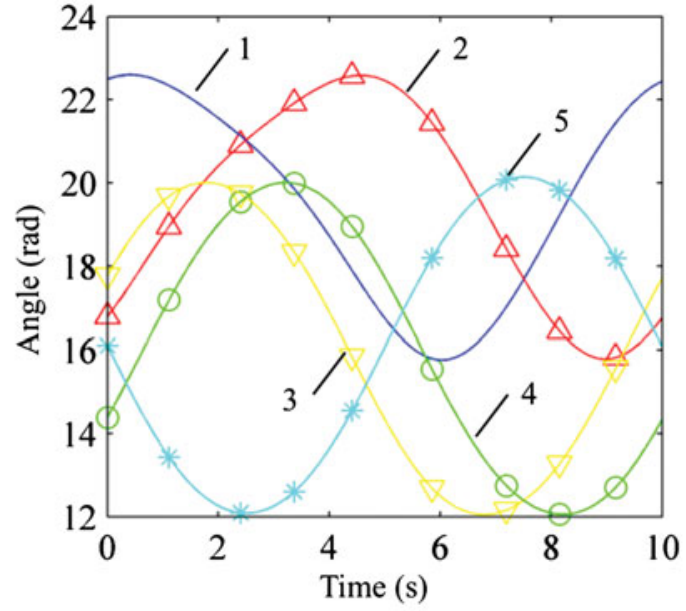

(b)

Fig. 16. Angle of $i$ th pulleys ( $i=1,2,3,4,5$ ). (a) Sliding mode control. (b) PD control.

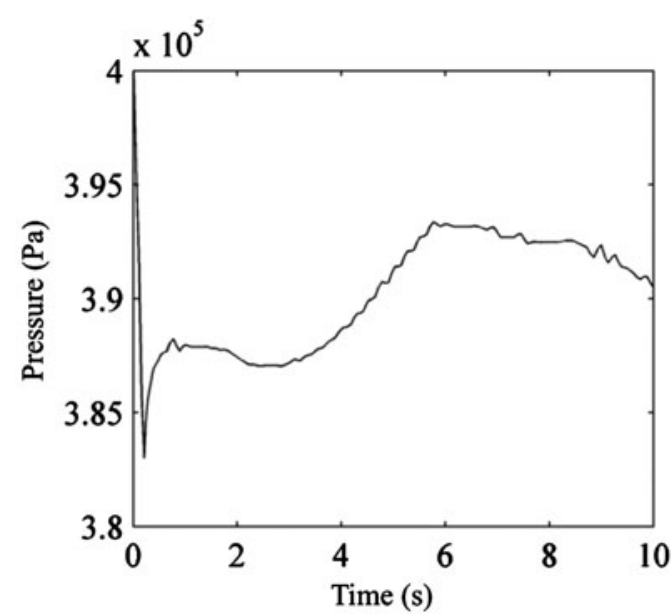

(a)

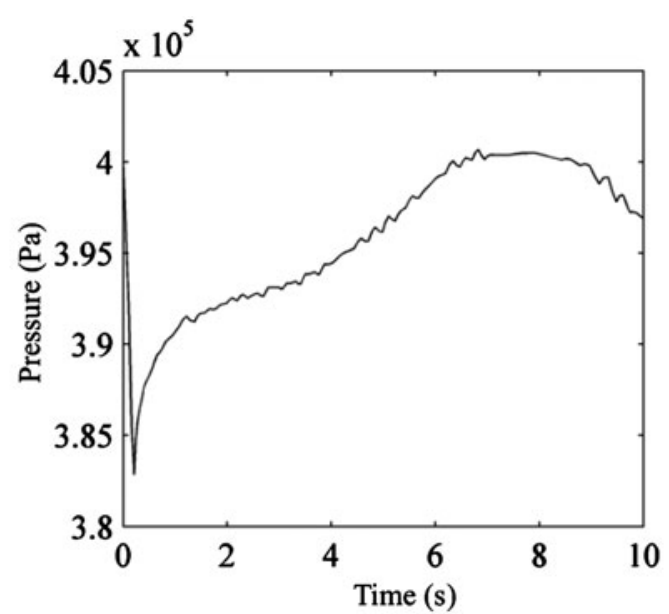

(b)

Fig. 17. Absolute pressure in the pneumatic muscle. (a) Sliding mode control. (b) PD control.

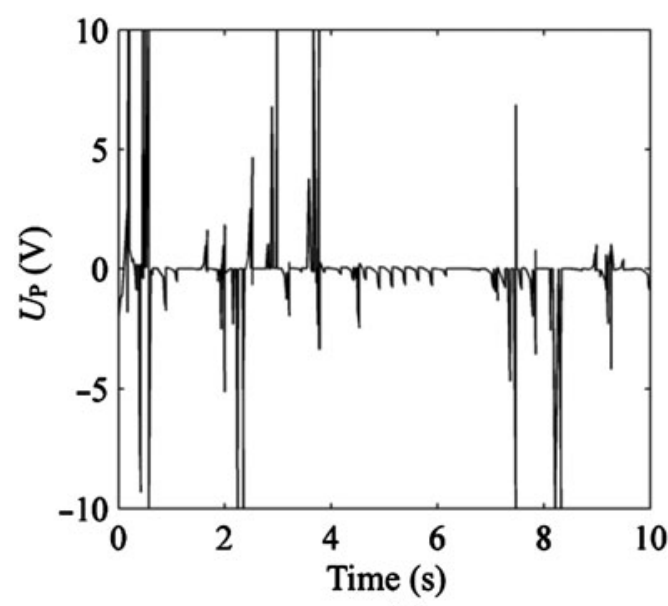

(a)

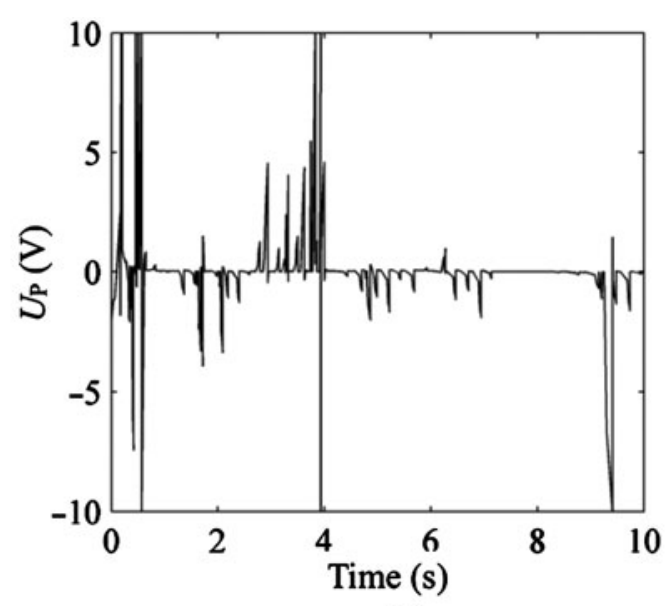

(b)

Fig. 18. Input voltage of the fast switching valve. (a) Sliding mode control. (b) PD control. 
Table III. Comparison in control performance of both controllers.

\begin{tabular}{lccc}
\hline Type & Sliding mode control $\left(e_{s}\right)$ & PD control $\left(e_{p}\right)$ & $\left(e_{s}-e_{p}\right) / e_{p}$ \\
\hline Maximum angle error & $5.5 \mathrm{e}^{-4}(\mathrm{rad})$ & $1.2 \mathrm{e}^{-3}(\mathrm{rad})$ & $54.17 \%$ \\
Maximum angle velocity error & $5.85 \mathrm{e}^{-4}(\mathrm{rad} / \mathrm{s})$ & $8.2 \mathrm{e}^{-4}(\mathrm{rad} / \mathrm{s})$ & $28.66 \%$ \\
Maximum length error & $5 \mathrm{e}^{-4}(\mathrm{~m})$ & $1 \mathrm{e}^{-3}(\mathrm{~m})$ & $50 \%$ \\
Maximum length change rate error & $5.7 \mathrm{e}^{-4}(\mathrm{~m} / \mathrm{s})$ & $8.4 \mathrm{e}^{-4}(\mathrm{~m} / \mathrm{s})$ & $43.14 \%$ \\
\hline
\end{tabular}

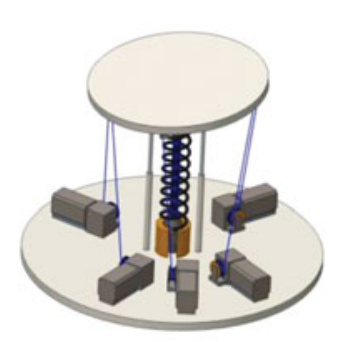

$t=1.6 \mathrm{~s}$

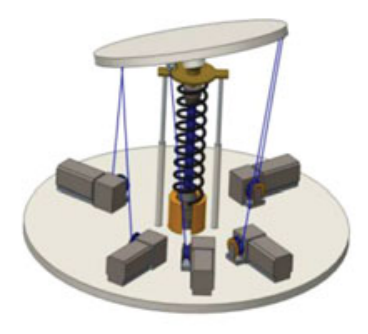

$t=3.3 \mathrm{~s}$

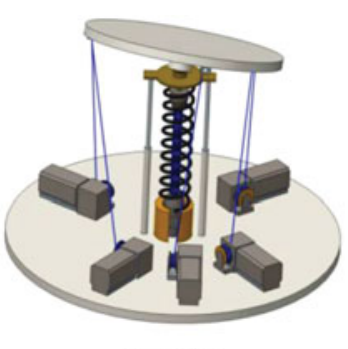

$t=6.6 \mathrm{~s}$

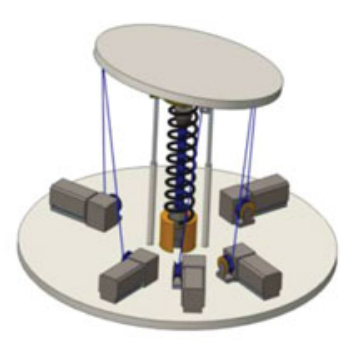

$t=8.3 \mathrm{~s}$

Fig. 19. Poses of the CPPPMS at different instants. $t=1.6 \mathrm{~s} . t=3.3 \mathrm{~s} . t=6.6 \mathrm{~s} . t=8.3 \mathrm{~s}$.

maximum length error for the sliding mode control is 50\% smaller than the PD control. In conclusion, the sliding model controller is more effective for the CPPPMS than that of the PD controller.

\section{Conclusion}

In this paper, a CPPPMS has been presented as an approach to imitating the ways in which the human neck works. According to the analysis, the following conclusions can be drawn:

1. The CPPPMS is a redundant driven mechanism with 4-DOF in terms of three rotations and one translation. Five cables and a pneumatic muscle are used to imitate neck muscles and spinal muscles, respectively. The CPPPMS can imitate the motion of human necks and can facilitate the in-depth understanding of this motion.

2. The CPPPMS has been optimized by carrying out the stiffness analysis. The optimized structure is similar to that of a human neck structure, which shows the effectiveness of the index based on the stiffness characteristics. The advantage of the index is that it can evaluate the mechanism with redundant actuators. Subsequently, the real workspace of the mechanism has been plotted by identifying the singular position, and shows that the real workspace of the CPPPMS is similar to that of a human neck.

3. The dynamic model of the CPPPMS consists of the dynamics of the Up-platform, the cables, and the pneumatic muscle active support. The inverse dynamics of the CPPPMS is an underdetermined equation due to the actuator redundancy. In order to obtain a unique solution to the equation, a performance index needs to be optimized and used as a constraint.

4. A sliding mode controller has been investigated to control the CPPPMS, and a PD controller has been proposed to provide a comparative study. The sliding mode controller is more effective in attacking mode uncertainties; however, jumps appear when the state variables go across the sliding manifold. In contrast, the PD controller cannot control the mechanism tracking the designed trajectory precisely due to the model uncertainties. Therefore, the sliding mode controller is more effective than the PD controller for the CPPPMS, and the CPPPMS provides feasible operation performances for operations under the sliding mode control.

5. The prototype verification of the designed CPPPMS and the installation of the control hardware are the topics for further research and are currently under investigation.

\section{Acknowledgements}

This work was supported by the National Natural Science Foundation of China (51575150), the Fundamental Research Funds for the Central Universities (2014HGCH0015), and China Scholarship 
Council (CSC). The authors appreciate the comments and valuable suggestions of anonymous referees and editors for improving the quality of the paper.

\section{Appendix A}

$$
\begin{gathered}
\boldsymbol{J}_{t}=\left[\begin{array}{cccc}
(-S \alpha S \beta C \gamma+C \alpha S \gamma) h_{2} & C \alpha C \beta C \gamma h_{2} & (-C \alpha S \beta S \gamma+S \alpha C \gamma) h_{2} & 0 \\
(C \alpha S \beta C \gamma+S \alpha S \gamma) h_{2} & S \alpha C \beta C \gamma h_{2} & (-S \alpha S \beta S \gamma-C \alpha C \gamma) h_{2} & 0 \\
0 & -S \beta C \gamma h_{2} & -C \beta S \gamma h_{2} & 1
\end{array}\right], \\
\boldsymbol{J}_{\tau}=\left[\begin{array}{cccc}
0 & -S \alpha & C \alpha C \beta & 0 \\
0 & C \alpha & S \alpha C \beta & 0 \\
1 & 0 & -S \beta & 0
\end{array}\right],
\end{gathered}
$$

where $S$ denotes sine and $C$ denotes cosine.

$$
\begin{aligned}
& \boldsymbol{J}=\boldsymbol{J}_{t}^{\mathrm{T}} m_{u} \boldsymbol{J}_{t}+\boldsymbol{J}_{r}^{\mathrm{T}} \boldsymbol{I}_{u} \boldsymbol{J}_{r}, \\
& \boldsymbol{\eta}=\boldsymbol{J}_{t}^{\mathrm{T}} m_{u} \dot{\boldsymbol{J}}_{t} \dot{\boldsymbol{Y}}+\boldsymbol{J}_{r}^{\mathrm{T}} \boldsymbol{I}_{u} \dot{\boldsymbol{J}}_{r} \dot{\boldsymbol{Y}}-\boldsymbol{J}_{t}^{\mathrm{T}} \boldsymbol{G}, \\
& \boldsymbol{H}=\boldsymbol{J}_{t}^{\mathrm{T}}\left[\begin{array}{llllll}
\boldsymbol{s}_{A B 1} & \boldsymbol{s}_{A B 2} & \boldsymbol{s}_{A B 3} & \boldsymbol{s}_{A B 4} & \boldsymbol{s}_{A B 5} & \boldsymbol{s}_{O C}
\end{array}\right] \\
& +\boldsymbol{J}_{r}^{\mathrm{T}}\left[\begin{array}{lllll}
\boldsymbol{s}_{A B 1} \times \boldsymbol{r}_{C B 1} & \boldsymbol{s}_{A B 2} \times \boldsymbol{r}_{C B 2} & \boldsymbol{s}_{A B 3} \times \boldsymbol{r}_{C B 3} & \boldsymbol{s}_{A B 4} \times \boldsymbol{r}_{C B 4} & \boldsymbol{s}_{A B 5} \times \boldsymbol{r}_{C B 5}
\end{array}\right],
\end{aligned}
$$

where $\boldsymbol{J}$ is the mass matrix, includes the mass and moment of inertia of the Up-platform, $\boldsymbol{\eta}$ includes the damping and the Coriolis acceleration of system, and $\boldsymbol{H}$ is a direction matrix, which indicates the direction of each external force and moment. All the matrices are described under the state variables coordinates.

\section{Appendix B}

$$
\begin{aligned}
\boldsymbol{\eta}_{f} & =-\boldsymbol{H}\left[\ldots, \eta_{f i}, \eta_{f p}\right]^{T}, \quad i=1,2,3,4,5 \\
\boldsymbol{H}_{f} & =\boldsymbol{H} \operatorname{diag}\left(\ldots, \boldsymbol{H}_{\mathrm{fai}}, \ldots, \boldsymbol{H}_{f p}\right), \quad i=1,2,3,4,5,
\end{aligned}
$$

where

$$
\begin{aligned}
& \eta_{f i}=\left\{\begin{array}{cc}
-\mu \dot{S}_{A B i}-E A_{a} & \text { if } R \theta_{i}-S_{A B i}>0 \\
0 & \text { if } R \theta_{i}-S_{A B i} \leq 0
\end{array}\right. \\
& \eta_{f p}=\left\{\begin{array}{cc}
\left(3 a h_{1}^{2}+b\right) P_{0}+\left(h_{1}-h_{0}\right) k_{s} & \text { if } 3 a h_{1}^{2}+b<0 \\
\left(h_{1}-h_{10}\right) k_{s} & \text { if } 3 a h_{1}^{2}+b \geq 0
\end{array}\right. \\
& H_{\text {fai }}= \begin{cases}\frac{E A_{a}}{S_{A B i}} \quad \text { if }-S_{A B i}+R \theta_{i}>0 \\
0 & \text { if }-S_{A B i}+R \theta_{i} \leq 0\end{cases} \\
& H_{f p}=\left\{\begin{array}{cc}
-\left(3 h_{1}^{2}+b\right) & \text { if } 3 a h_{1}^{2}+b<0 \\
0 & \text { if } 3 a h_{1}^{2}+b \geq 0
\end{array}\right.
\end{aligned}
$$




\section{References}

1. A. Kiapour, A. M. Kiapour, V. Kaul, C. E. Quatman, S. C. Wordeman, T. E. Hewett and V. K. Goel, "Finite element model of the knee for investigation of injury mechanisms: Development and validation," $J$. Biomech. Eng. 136(1), 011002.1-011002.14 (2014).

2. K. W. Choi and A. M. Schmitz, "Co-simulation of neuromuscular dynamics and knee mechanics during human walking," J. Biomech. Eng. 136(1), 021033.1-021033.8 (2014).

3. A. A. White and M. M. Panjabi, Clinical Biomechanics of the Spine, (JB Lippincott, Philadelphian, 1990), vol. 2, pp. 108-112.

4. Y. Sakagami, R. Watanabe, C. Aoyama, S. Matsunaga, N. Higaki and K. Fujimura, "The Intelligent ASIMO: System Overview and Integration," IEEE/RSJ International Conference on, Intelligent Robots and Systems, EPFL, Lausanne, Switzerland (2002), vol. 3, pp. 2478-2483.

5. K. Kaneko, K. Harada, F. Kanehiro, G. Miyamori and K. Akachi, "Humanoid Robot Hrp-3," IEEE/RSJ International Conference on, Intelligent Robots and Systems IROS 2008, Nice, France (2008) pp. 24712478.

6. Q. Huang, Z. Peng, W. Zhang, L. Zhang and K. Li, "Design of Humanoid Complicated Dynamic Motion based on Human Motion Capture," IEEE/RSJ International Conference on, Intelligent Robots and Systems, Alberta Canada (2005) pp. 3536-3541.

7. S. Lohmeier, T. Buschmann and H. Ulbrich, "Humanoid Robot Lola," IEEE International Conference on, Robotics and Automation ICRA'09, Kobe, Japan (2009) pp. 775-780.

8. J. Han, S. Zeng, K. Tham, M. Badgero and J. Weng, "Dav: A Humanoid Robot Platform for Autonomous Mental Development," Proceedings of the 2nd International Conference on Development and Learning, Cambridge, Massachusetts, USA (2002) pp. 73-81.

9. F. Guenter, L. Roos, A. Guignard and A. G. Billard, "Design of a Biomimetic Upper Body for the Humanoid Robot Robota," 5th IEEE-RAS International Conference on, Humanoid Robots, Tsukuba, Japan (2005) pp. $56-61$.

10. T. Asfour, P. Azad, N. Vahrenkamp, K. Regenstein, A. Bierbaum, K. Welke, J. Schroder and R. Dillmann, "Toward humanoid manipulation in human-centred environments," Robot. Auton. Syst. 56(1), 54-65 (2008).

11. G. Carbone, H.-O. Lim, A. Takanishi and M. Ceccarelli, "Stiffness analysis of biped humanoid robot WABIANRIV," Mech. Mach. Theory 41(1), 17-40 (2006).

12. O. Holland and R. Knight, "The Anthropomimetic Principle," Proceedings of the AISB06 Symposium on Biologically Inspired Robotics, Bristol, UK (2006) pp. 1-8.

13. L. Jamone, G. Metta, F. Nori and G. Sandini, "James: A Humanoid Robot Acting Over An Unstructured World," 6th IEEE-RAS International Conference on Humanoid Robots, Genoa, Italy (2006) pp. 143-150.

14. F. Nori, L. Jamone, G. Sandini and G. Metta, "Accurate Control of a Human-Like Tendon-Driven Neck," 7th IEEE-RAS International Conference on Humanoid Robots, Pittsburgh, Pennsylvania (2007) pp. 371-378.

15. B. Gao, H. Song, J. Zhao, S. Guo, L. Sun and Y. Tang, "Inverse kinematics and workspace analysis of a cable-driven parallel robot with a spring spine," Mech. Mach. Theory 76(1), 56-69 (2014).

16. S. H. Lee and D. Terzopoulos, "Heads up!: Biomechanical modeling and neuromuscular control of the neck," ACM Trans. Graph. 25(3), 1188-1198 (2006).

17. K. Liem, A. Kecskeméthy and J. Merlet, "Hexaspine: A Parallel Platform for Physical Cervical Spine Simulation-Design and Interval-based Verification," Proceedings of the 12th World Congress in Mechanism and Machine Science, BESANCON - FRANCE (2007) pp. 17-21.

18. B. Zi, Z. C. Zhu and J. L. Du, 2011, "Analysis and control of the cable-supporting system including actuator dynamics," Control Eng. Pract. 19(5), 491-501.

19. B. Zi, B. Y. Duan, J. L. Du and H. Bao, "Dynamic modeling and active control of a cable-suspended parallel robot," Mechatronics 18(1), 1-12 (2008).

20. Y. B. Bedoustani, H. D. Taghirad and M. M. Aref, "Dynamics Analysis of a Redundant Parallel Manipulator Driven by Elastic Cables," 10th International Conference on, Control, Automation, Robotics and Vision ICARCV 2008, Hanoi, Vietnam (2008) pp. 536-542.

21. M. Hiller, S. Fang, S. Mielczarek, R. Verhoeven and D. Franitza, "Design, analysis and realization of tendon-based parallel manipulators," Mech. Mach. Theory 40(4), 429-445 (2005).

22. X. Zhao and B. Zi, "Design and analysis of a pneumatic muscle driven parallel mechanism for imitating human pelvis," Proc. Inst. Mech. Eng., Part C: Journal of Mechanical Engineering Science, 228(4), 723-741 (2014).

23. C. Chou and B. Hannaford, "Measurement and modeling of mckibben pneumatic artificial muscles," IEEE Trans. Robot. Autom. 12(1), 90-102 (1996).

24. P. K. Jamwal, S. Q. Xie, S. Hussain and J. G. Parsons, "An adaptive wearable parallel robot for the treatment of ankle injuries," IEEE/ASME Trans. Mechatronics 19(1), 64-75 (2012).

25. X. Zhu, G. Tao, B. Yao and J. Cao, "Adaptive robust posture control of parallel manipulator driven by pneumatic muscles with redundancy," IEEE/ASME Trans. Mechatronics 13(4), 441-450 (2008).

26. K. Xing, Y. Wang, Q. Zhu and H. Zhou, "Modeling and control of mckibben artificial muscle enhanced with echo state networks," Control Eng. Pract. 20(5), 477-488 (2012).

27. L. D. Khoa, D. Q. Truong and K. K. Ahn, "Synchronization controller for a 3-R planar parallel pneumatic artificial muscle robot using modified ANFIS algorithm," Mechatronics 23(4), 462-479 (2013).

28. Z. Man, A. P. Paplinski and H. R. Wu, "A robust MIMO terminal sliding mode control scheme for rigid robotic manipulators," IEEE Trans. Autom. Control 39(12), 2464-2469 (1994). 
29. F. Yong, X. Yu and Z. Man, "Non-singular terminal sliding mode control of rigid manipulators," Automatica 38(12), 2159-2167 (2002).

30. X. Shen, "Nonlinear model-based control of pneumatic artificial muscle servo systems," Control Eng. Pract. 18(3), 311-317 (2010).

31. G. L. Shi and W. Shen, "Hybrid control of a parallel platform based on pneumatic artificial muscles combining sliding mode controller and adaptive fuzzy CMAC," Control Eng. Pract. 21(1), 76-86 (2013).

32. R. S. Ball, A Treatise on the Theory of Screws, Cambridge, UK (Cambridge University Press, 1990).

33. H. Liu, T. Huang and D. G. Chetwynd, "A method to formulate a dimensionally homogeneous Jacobian of parallel manipulators," IEEE Trans. Robot. 27(1), 150-156 (2011).

34. N. Ciblak and H. Lipkin, "Asymmetric Cartesian Stiffness for the Modeling of Compliant Robotic Systems," Proceedings of the 23rd Biennial ASME Mechanisms Conference, Minneapolis, MN (1994) pp. 197-204.

35. N. Ciblak and H. Lipkin, "Synthesis of Cartesian Stiffness for Robotic Applications," Proceedings of the IEEE International Conference on, Robotics and Automation, Detroit, Michigan (1999), vol. 3, pp. 2147-2152.

36. C. C. Gosselin and J. J. Angeles, "A Global Performance Index for the Kinematic Optimization of Robotic Manipulators," J. Mech. Des. 113(3), 220-226 (1991).

37. G. S. Chirikjian and A. B. Kyatkin, Engineering Applications of Noncommutative Harmonic Analysis: With Emphasis on Rotation and Motion Groups, Boca Raton, Florida (CRC Press 2000).

38. C. M. Gosselin and M. Grenier, "On the determination of the force distribution in overconstrained cabledriven parallel mechanisms," Meccanica 46(1), 3-15 (2011).

39. P. R. Ouyang, W. J. Zhang and F. X. Wu, "Nonlinear PD Control for Trajectory Tracking with Consideration of the Design for Control Methodology," Proceedings of the IEEE International Conference on, Robotics and Automation ICRA'02, Washington, D.C. (2002) pp. 4126-4131.

40. F. X. Wu, W. J. Zhang, Q. Li and P. R. Ouyang, "Integrated design and PD control of high-speed closed-loop mechanisms," J. Dyn. Syst. Meas. Control 124(4), 522-528 (2002). 